\title{
Multi-bump solutions in a neural field model with external inputs
}

\author{
Flora Ferreira ${ }^{a}$, Wolfram Erlhagen ${ }^{\mathrm{a}, *}$, Estela Bicho ${ }^{\mathrm{b}}$ \\ ${ }^{\text {a }}$ Centre of Mathematics, University of Minho, Portugal \\ ${ }^{\mathrm{b}}$ Centre Algoritmi, University of Minho, Portugal
}

\section{H I G H L I G H T S}

- Stable N-bump solutions in a field with $\mathrm{N}$ localized inputs are analyzed.

- Conditions for the shape of the input distribution ensure the existence.

- The effect of spatial interactions in a continuous attractor network is discussed.

- For a given finite field interval, the maximum number of bumps can be determined.

- The results are discussed in terms of a precise spatial memory mechanism.

\section{A R T I C L E I N F O}

\section{Article history:}

Received 7 July 2015

Accepted 28 January 2016

Available online $\mathrm{xxxx}$

Communicated by S. Coombes

\section{Keywords:}

Pattern formation

Working memory

Integro-differential equation

Transient external input

Persistent neural population activity

\begin{abstract}
A B S T R A C T
We study the conditions for the formation of multiple regions of high activity or "bumps" in a onedimensional, homogeneous neural field with localized inputs. Stable multi-bump solutions of the integrodifferential equation have been proposed as a model of a neural population representation of remembered external stimuli. We apply a class of oscillatory coupling functions and first derive criteria to the input width and distance, which relate to the synaptic couplings that guarantee the existence and stability of one and two regions of high activity. These input-induced patterns are attracted by the corresponding stable one-bump and two-bump solutions when the input is removed. We then extend our analytical and numerical investigation to $N$-bump solutions showing that the constraints on the input shape derived for the two-bump case can be exploited to generate a memory of $N>2$ localized inputs. We discuss the pattern formation process when either the conditions on the input shape are violated or when the spatial ranges of the excitatory and inhibitory connections are changed. An important aspect for applications is that the theoretical findings allow us to determine for a given coupling function the maximum number of localized inputs that can be stored in a given finite interval.
\end{abstract}

(C) 2016 Elsevier B.V. All rights reserved.

\section{Introduction}

In recent years, the analysis of pattern formation in neural field models of cortical tissue has been a very active area of research in the emerging field of mathematical neuroscience (for reviews see $[1,2])$. These models take the form of nonlinear integro-differential equations on a spatially extended domain. Their dynamics is known to support a large variety of coherent structures observed in neural population activity including stationary "bumps" of localized excitation, as well as spatial or spatiotemporal oscillation patterns, and traveling waves. The mathematical analysis of field models has provided new insight into the

\footnotetext{
* Corresponding author.

E-mail address: wolfram.erlhagen@math.uminho.pt (W. Erlhagen).
}

conditions of excitatory and inhibitory interactions within neural populations, which ensure the existence and stability of these patterns. In a complementary line of research, bump attractors of neural population dynamics have been applied as models of cognitive processes such as visual attention, motor planning, decision making and working memory in biological and artificial agents ([3-10]; for reviews see [11,12]). In these applications, the neural fields are defined over continuous metric dimensions such as movement direction, retinal position, color or tone pitch. Due to the assumed translation invariance of neural interactions, the field supports a spatial continuum of persistent localized activity patterns known as a "continuous attractor" [13]. Transient input from external sensors representing information about a specific value along the coded dimension defines the field location where a self-stabilized bump evolves. While the peak position may serve a memory function in the case of a single cue, the situation is more complex when two or more localized stimuli are applied simultaneously. 
Since bumps are neutrally stable to perturbations in their position $[14,13]$, the spatial interactions between several bumps in the field mediated by the recurrent architecture of the local network may lead to changes in their position. These interactions may thus result in a stationary pattern for which the number and positions of peaks do not match the number and locations of the external stimuli. As was previously qualitatively discussed by Amari [14] for the case of a connection function of "Mexican-hat" type (i.e., local excitation and surround inhibition), depending on the precise shape of the spatial couplings two input-induced local excitations separated by a certain distance may repel or attract each other. In the case of attraction, the two regions of excitation may eventually combine into a single bump at an intermediate position between the stimulated sites. While this behavior can be exploited to model for instance experimental findings in certain oculomotor decision tasks [8], it is obviously inadequate for the representation of a short-term maintenance of the two inputs (but see [15] for behavioral evidence of an attraction effect of neighboring items in working memory). In applications requiring the storage of a series of distinct cues with high precision $[10,16]$, the impact of the mutual interactions between input-induced local excitations on the evolving memory representation should be minimal. Furthermore, since a single localized input may activate more than one bump, depending on its width, it is not only the relative position of the individual inputs that matters, but also their very size.

The main goal of the present study is to extend previous formal arguments for the existence and stability of multi-bump solutions in spatially homogeneous fields without external stimuli $[17,18]$ to the case of a field dynamics in the presence of one or more localized inputs. More specifically, we establish conditions for the width and relative distance of the external stimuli, in terms of the coupling function, in order to ensure a precise multiitem memory representation in a continuous attractor network. The rigorous analysis allows us to better understand the pattern formation process when the conditions on the shape of the input distribution are violated. In particular, we study, both analytically and in numerical simulations, how changes in the spatial ranges of the excitatory and inhibitory couplings (e.g., during development and learning [15,7]) affect the field response to a given input distribution.

We investigate a particular formulation of a dynamic field first introduced and analyzed by Amari [14] and subsequently used in many applications $[12,11]$ :

$$
\begin{aligned}
\frac{\partial u(x, t)}{\partial t}= & -u(x, t)+\int_{-\infty}^{\infty} w(x-y) f(u(y, t)) d y \\
& -h+S(x, t) .
\end{aligned}
$$

Here, $u(x, t)$ represents the average level of activity (e.g., voltage) of a neuron at spatial position $x$ and time $t$ along a onedimensional infinite domain. The nonlinear function $f(u)$ defines the firing rate of a neuron with activity $u$. The function $w(x)$ describes the coupling strength with neighboring neurons $y$, which is assumed to depend on the distance only, that is, $w(x \mid y)=w(x-y)$. The term $S(x, t)$ represents a transient external input with a spatial structure, whereas $-h<0$ denotes a constant inhibitory input applied uniformly to the entire field. This global inhibition defines a homogeneous "resting" state for a neural field with $S(x)=0 \forall x$.

For the special choice of a Heaviside firing function and synaptic couplings of Mexican-hat type, Amari fully analyzed the existence and stability properties of a single-bump stationary solution of Eq. (1) with a unimodal and symmetric input distribution [14,19]. Since a stable bump co-exists with the stable resting state, a sufficiently strong transient input may switch between the two states, thus implementing a memory function. However, analytical and numerical studies have shown that a coupling function of
Mexican-hat shape, which changes sign exactly once in the interval $(0, \infty)$, does not generally support a stable pattern of two or more regions of high excitation ([18], but see the discussion in [20,21] for large distances between bumps). In the present study we therefore apply a class of oscillatory coupling functions, previously introduced by Laing and colleagues [17], with an infinite number of positive zeros in $(0, \infty)$. The authors showed numerical evidence for the existence of multiple stable bumps in a homogeneous field without external input.

The paper is organized as follows: in Section 2 we review relevant results of previous studies on the existence and stability of one-bump and two-bump solutions and provide a detailed mathematical description of the model assumptions. In Section 3, we generalize Amari's analysis of a single bump in the presence of a localized input; for the class of oscillatory coupling functions, we determine conditions for the shape of the input $S(x)$ as well as for the global inhibition $h$, which guarantees the evolution of a stable region of local excitation. In Section 4, we extend the analysis for a specific value of $h$ to the case of two-bump solutions of Eq. (1) with a bimodal, symmetric input. Based on the insight obtained from the analysis of the two-bump activation patterns, Section 5 presents analytical and numerical work on the existence and stability of input-induced $N$-bump solutions for $N \geq 2$. A brief summary of our results, as well as an outlook on future research, is presented in Section 6. In order to allow readers from applied areas to focus on the main findings, we present the mathematical proof of all theorems stated in the main text in Appendix A. The scheme for the numerical integration of Eq. (1) used in the simulations of the field model with external input is presented in Appendix B.

\section{Model details and problem statement}

We study the existence and the stability of steady state solutions of (1), i.e. solutions defined by

$u(x)=\int_{-\infty}^{\infty} w(x-y) f(u(y, t)) d y-h+S(x)$.

In order to simplify the mathematical treatment of single-bump solutions, Amari chose the Heaviside activation function

$f(u)=H_{0}(u)= \begin{cases}0, & u \leq 0 \\ 1, & u>0,\end{cases}$

instead of a continuous function of sigmoidal shape. The main advantage is that the dynamics of a local excitation pattern can be understood by analyzing the much simpler motion equations of its boundaries. For the lateral connections between neurons, Amari used a coupling function $w(x)$ in which excitation dominates over smaller distances and inhibition over larger ones. Such a connectivity of "lateral inhibition" type satisfies the following properties:

$\left(\mathrm{H}_{1}\right) w(x)$ is symmetric, i.e., $w(-x)=w(x)$ for all $x \in \mathbb{R}$.

$\left(\mathrm{H}_{2}\right) w$ is both continuous and integrable on $\mathbb{R}$.

$\left(\mathrm{H}_{3}\right) w(x)>0$ on an interval $(0, \bar{x}), w(x)<0$ on $(\bar{x}, \infty)$ and $w(\bar{x})=0$.

$\left(\mathrm{H}_{4}\right) w(x)$ is decreasing on $(0, \bar{x}]$.

A concrete example of a coupling function satisfying $\left(\mathrm{H}_{1}\right)-\left(\mathrm{H}_{4}\right)$ is a "Mexican-hat" function given by

$w(x)=M e^{-m|x|}-N e^{-n|x|}$,

where $M>N>0$ and $m>n>0$ (Fig. 1, left).

Motivated by neuron labeling studies showing that the spatial coupling between groups of neurons in the prefrontal cortex forms approximate periodic stripes [22], Laing and colleagues [17,18] 

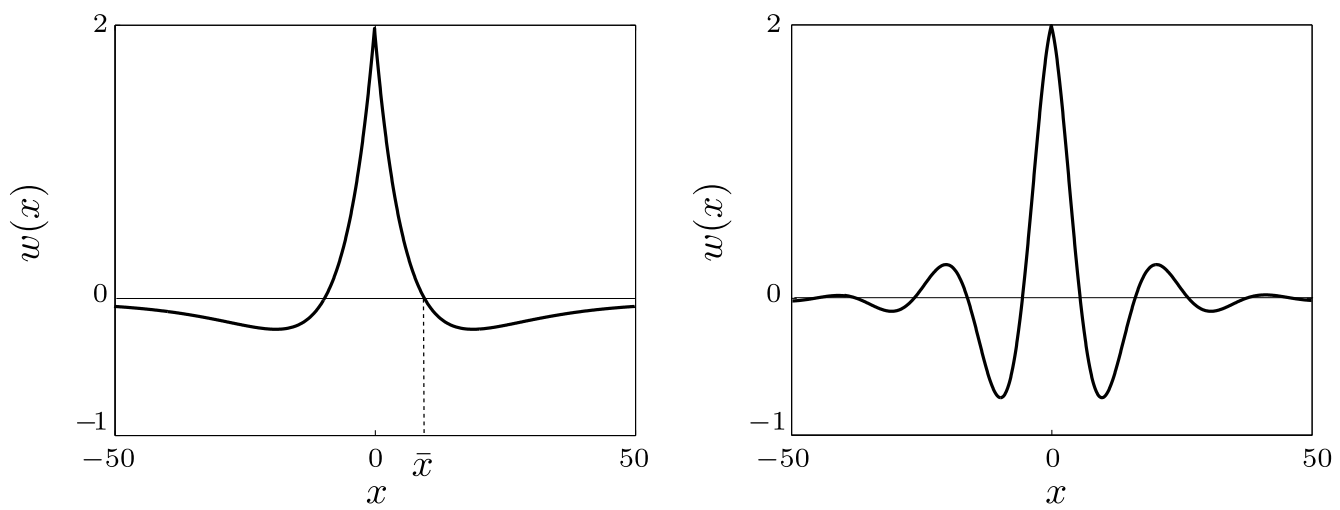

Fig. 1. Coupling functions. Left: $w(x)$ defined by (4) with $M=4, N=2, m=0.15$ and $n=0.08$. Right: $w(x)$ defined by (6) with $A=2, k=0.1$ and $\alpha=0.3$.

proposed a class of coupling functions with oscillatory rather than monotonic decay

$w(x)=e^{-k|x|}(k \sin |x|+\cos (x))$,

where the parameter $k>0$ controls the rate at which the oscillations of $w(x)$ decay in relation to distance.

With the multi-item working memory application in mind, in this paper we study the stimulus-induced formation of multibumps using a modified version of this class of intra-field couplings

$w(x)=A e^{-k|x|}(k \sin |\alpha x|+\cos (\alpha x))$,

where the parameters $A>0$ and $k<\alpha \leq 1$ are added to control the amplitude and the zero crossings, of $w(x)$, respectively (Fig. 1, right). The larger the value $\alpha$, the smaller is the distance between consecutive zeros. Since $\alpha$ determines the spatial ranges of lateral excitation and lateral inhibition in the field, changes in this model parameter can be used to control the number of bumps that may exist in a given finite interval, that is, the spatial resolution of the memory representation (see Section 5 ).

In addition to $\left(\mathrm{H}_{1}\right)$ and $\left(\mathrm{H}_{2}\right)$, the class of coupling functions (6) satisfies the following properties:

$\left(\mathrm{H}_{5}\right) w(x)$ is an oscillatory function that tends to zero as $x \rightarrow \pm \infty$.

$\left(\mathrm{H}_{6}\right) w(0)>0$, and $w$ has infinite positive zeros at values $z_{n}$, $n \in \mathbb{N}$.

In order to prove the existence of one-bump solutions, Amari defined the function

$W(x)=\int_{0}^{x} w(y) d y$

and the related quantities $W_{m}=\max _{x>0} W(x)$ and $W_{\infty}=$ $\lim _{x \rightarrow \infty} W(x)$. It follows from conditions $\left(\mathrm{H}_{1}\right)$ and $\left(\mathrm{H}_{2}\right)$ that $W(x)$ is continuously differentiable and odd, and that $W_{\infty}$ is finite.

A coupling function given by (6) has an infinite number of positive zeros in $(0, \infty)$ at values

$z_{n}=-\frac{\arctan \left(\frac{1}{k}\right)}{\alpha}+\frac{n \pi}{\alpha}$ for all $n \in \mathbb{N}$,

and the integral of $w(x)$ for $x \geq 0$ is given by

$W(x)=-p_{1}\left(e^{-k x}\left(p_{3} \sin (\alpha x)+p_{2} \cos (\alpha x)\right)-p_{2}\right)$

where $p_{1}=\frac{A}{k^{2}+\alpha^{2}}, p_{2}=\alpha k+k$ and $p_{3}=k^{2}-\alpha$. Note that the zeros $z_{n}$ of $w(x)$ define the local maxima ( $n$ odd) and the local minima ( $n$ even) of $W(x)$.

Following Amari's analysis [19], in Section 3 we consider that a single stationary input $S(x)$ centered at $x=0$ has a unimodal and symmetric shape. More specifically, the input presents the following properties
$\left(\mathrm{SH}_{1}\right) S(x)$ is continuous on $\mathbb{R}$ and symmetric in relation to the center, i.e., $S(-x)=S(x)$ for all $x \in \mathbb{R}$.

$\left(\mathrm{SH}_{2}\right) S(x)>0$ on an interval $(0, \bar{x}), S(x)<0$ on $(\bar{x}, \infty)$, and $S(\bar{x})=0$.

$\left(\mathrm{SH}_{3}\right) S(x)$ decreases on $(0, \infty)$.

A concrete example that was used in our numerical investigations is

$S_{1 b}(x)=S_{s} e^{\left(-\frac{x^{2}}{2 \sigma^{2}}\right)}-S_{i}$,

where $S_{s}>0$ and $\sigma>0$ describe the amplitude and the standard deviation of a Gaussian function, respectively, and $S_{i}>0$ is a constant. Note that for the analysis of the pattern formation process, the constant negative part could also be integrated in the global inhibition parameter $h$ of the field equation. We use it in the definition of the Gaussian input to define a finite width $a_{s}$ of the positive input range $\left(-\frac{a_{s}}{2}, \frac{a_{s}}{2}\right)$. In the following we will refer to an external input satisfying $\left(\mathrm{SH}_{1}\right),\left(\mathrm{SH}_{2}\right)$ and $\left(\mathrm{SH}_{3}\right)$ as $\mathrm{S}_{1 b}(x)$.

If Eq. (2) has a one-bump solution whose region of excitation $u(x)>0$ is the interval $R[u]=\left(-\frac{a}{2}, \frac{a}{2}\right)$, then the solution satisfies [19]

$$
\begin{aligned}
u(x) & =\int_{-\frac{a}{2}}^{\frac{a}{2}} w(x-y) d y-h+S(x) \\
& =W\left(x+\frac{a}{2}\right)-W\left(x-\frac{a}{2}\right)-h+S_{1 b}(x) .
\end{aligned}
$$

At point $x=\frac{a}{2}$, since $W(x)$ is odd and $u\left(-\frac{a}{2}\right)=u\left(\frac{a}{2}\right)=0$, Eq. (11) is reduced to

$W(a)=h-S_{1 b}\left(\frac{a}{2}\right)$.

In turn, if $a>0$ and $h>0$ satisfy (12), then

$u(x)=W\left(x+\frac{a}{2}\right)-W\left(x-\frac{a}{2}\right)-h+S_{1 b}(x)$

defines a one-bump solution of Eq. (2) with $R[u]=\left(-\frac{a}{2}, \frac{a}{2}\right)$.

So to analyze bump stability, Amari considered the boundary points of the local excitation and derived ordinary differential equations for their motions assuming small perturbations. The dynamics for the boundaries can then be reduced to a simple dynamics of the length $a(t)$ of the excited region. Considering the variational equation, one can conclude that a one-bump solution of Eq. (1) with localized input is stable if and only if

$\frac{d W}{d a}(a)=w(a)+\frac{1}{2} S_{1 b}^{\prime}\left(\frac{a}{2}\right)<0$,

where $S_{1 b}^{\prime}\left(\frac{a}{2}\right)$ denotes the derivative of the external input at $x=\frac{a}{2}$. Eq. (12) supports the co-existing of two single bumps that differ in 
their width. Amari showed that the large bump is always stable and the small bump is always unstable. In Section 3, we generalize Amari's analysis for a field of lateral-inhibition type and derive sufficient conditions on $h$ and $S_{1 b}$ for the general class of coupling functions given by (6), which guarantee the existence of a stable one-bump solution.

For a field without external input, Laing and Troy [18] extended the analysis of the Amari model to the case of two-bump solutions. They defined a two-bump pattern as a solution of Eq. (1) whose region of suprathreshold excitation is the union of two disjoint, finite and open intervals, that is, $R[u]=(0, a) \cup(b, c)$. Thus, a steady state solution $u(x)$ is called a two-bump solution if there are values $0<a<b<c<\infty$ such that

$$
\begin{cases}u>0 & \text { on }(0, a) \cup(b, c), \\ u(0)=u(a)=u(b)=u(c)=0, & \text { otherwise. }\end{cases}
$$

Specifically, Laing and Troy were interested in the symmetric case where the bumps have equal width, that is, $c=a+b$.

In keeping with our basic idea of a stable pattern that represents two instances of a certain stimulus dimension, we assume for the analysis that the external input $S(x)$ is of bimodal and symmetric shape, centered at $x=0$. It thus satisfies $\left(\mathrm{SH}_{1}\right)$ and the following properties:

$$
\left(\mathrm{SH}_{4}\right) S(x)>0 \text { on }\left(\overline{x_{1}}, \overline{x_{2}}\right), S(x)<0 \text { on }\left(0, \overline{x_{1}}\right) \cup\left(\overline{x_{2}}, \infty\right) \text { and }
$$
$S\left(\overline{x_{1}}\right)=S\left(\overline{x_{2}}\right)=0$.

$\left(\mathrm{SH}_{5}\right) S(x)$ is increasing on $\left(0, \frac{\overline{x_{2}}-\overline{x_{1}}}{2}\right)$, and is decreasing on $\left(\frac{\overline{x_{2}}-\overline{x_{1}}}{2}, \infty\right)$.

In the following, we will refer to a bimodal input satisfying $\left(\mathrm{SH}_{1}\right),\left(\mathrm{SH}_{4}\right)$ and $\left(\mathrm{SH}_{5}\right)$ as $\mathrm{S}_{2 b}(x)$.

We recall that a two-bump equal-width stationary solution, positive only over $\left(-\frac{b+a}{2},-\frac{b-a}{2}\right) \cup\left(\frac{b-a}{2}, \frac{b+a}{2}\right)$, is of the form:

$$
\begin{aligned}
u(x)= & W\left(x+\frac{b+a}{2}\right)-W\left(x+\frac{b-a}{2}\right)+W\left(x-\frac{b-a}{2}\right) \\
& -W\left(x-\frac{b+a}{2}\right)-h+S_{2 b}(x) .
\end{aligned}
$$

Consequently, since $u\left(-\frac{b+a}{2}\right)=u\left(-\frac{b-a}{2}\right)=u\left(\frac{b-a}{2}\right)=$ $u\left(\frac{b+a}{2}\right)=0$, and $W(x)$ is odd, the necessary conditions for the existence of such solutions are

$W(a)+W(b)-W(b-a)-h+S_{2 b}\left(\frac{b-a}{2}\right)=0$,

and

$W(a)-W(b)+W(a+b)-h+S_{2 b}\left(\frac{b+a}{2}\right)=0$.

For the case $S(x)=0 \forall x$, it was shown in [18] that there exists an interval of values for $a$ and corresponding values for $b>a$, so that the necessary condition for the existence of a two-bump solution of equal width, $2 W(b)-W(a+b)-W(b-a)=0$, is satisfied. However, as stressed in [18,23], it is difficult (i) to determine the sign of $h$, and (ii) to verify that the solution presents the correct positive ranges of excitation for these values of $a$ and $b$. Murdock et al. [23] addressed problem (i) by imposing appropriate conditions on a Mexican-hat coupling function that ensure $h \geq 0$. Assuming that $h=|W(p)|$ with $p \in\{a, b, c\}$, they demonstrated the existence of parameter values which satisfy conditions (17) and (18) for the case $S(x)=0 \forall x$. To emphasize that the positive ranges of solutions discussed in the literature are only verified numerically, they introduced the following definition of a quasisolution.
Definition 1. Given a triple of positive numbers, $\tau=(a, b, c)$ with $a<b<c$, and $h \geq 0$, the function $u_{\tau}(x)=W(x)-W(x-$ $a)+W(x-b)-W(x-c)-h$ is defined. If $p \in\{a, b, c\}, h=|W(p)|$, and $u_{\tau}$ satisfies $u_{\tau}(0)=u_{\tau}(a)=u_{\tau}(b)=u_{\tau}(c)=0$, then $u_{\tau}$ is said to be a $p$-quasi-solution of (1).

For a Mexican-hat coupling function $w(x)$ with $W_{\infty} \geq 0$, the authors proved the non-existence of a $a$-quasi-solution and the existence of both a $b$-quasi-solution and a $c$-quasi-solution [23]. A major challenge is to determine specific values for the width $a$ and the distance $b$ from the generic properties of $w(x)$, which satisfy the two conditions (17) and (18). In Section 4, this problem is addressed for the general class of coupling functions (6). We show that specific values of $a$ and $b$ exist if $h=|W(a)|$.

In order to analyze the stability of a two-bump equal-width solution for the case $S(x)=0$, Laing and Troy [18] followed Amari's approach [14] and derived a system of motion equations for $a(t)$ and $b(t)$ for which a linear stability analysis can be performed. The conditions

$$
\begin{aligned}
& w(a)<0, \quad w(b)>0, \\
& w(b-a)<0, \quad w(a+b)<0,
\end{aligned}
$$

ensure that the triple $(a, b, a+b)$ defines a stable solution with regard to perturbations that preserve the equal-width condition. For a Mexican-hat coupling function (4), an extensive study of the trace and the determinant of the Jacobian matrix of the linearized system, as functions of the various coupling parameters, showed that two-bump solutions do indeed exist but are unstable. This conclusion was confirmed for general perturbations of the steadystate solution in [23] by using an approach connected to the idea of the Evans function [21]. However, relaxing the hypotheses on $w(x)$ by assuming exactly three positive zeros proved to be sufficient in order to guarantee the co-existence of both stable and unstable two-bump solutions [18].

For a field with an external input satisfying $\left(\mathrm{SH}_{1}\right),\left(\mathrm{SH}_{4}\right)$ and $\left(\mathrm{SH}_{5}\right)$, and in addition to conditions (19), the necessary conditions for linear stability include also conditions for the input derivative

$S_{2 b}^{\prime}\left(\frac{b-a}{2}\right)>0$ and $S_{2 b}^{\prime}\left(\frac{a+b}{2}\right)<0$.

In Section 4, we generalize the analysis of the one-bump case and derive sufficient conditions for the shape of $S_{2 b}(x)$, which depend on the zeros of the coupling function (6), that guarantee the existence and stability of a family of two-bump patterns.

In order to analyze solutions with multiple local excitations, Laing and colleagues [17] introduced the class of oscillatory coupling functions (5). A solution $u(x)$ of Eq. (2) is called an $N$ bump solution if there are values $a_{0}<a_{1}<a_{2}<a_{3}<\cdots<$ $a_{2 N-1}$, so that

$\left\{\begin{array}{l}u>0 \text { on }\left(a_{0}, a_{1}\right) \cup \cdots \cup\left(a_{2 N-2}, a_{2 N-1}\right), \\ u\left(a_{0}\right)=u\left(a_{1}\right)=\cdots=u\left(a_{2 N-1}\right)=0, \\ u<0 \text { otherwise. }\end{array}\right.$

The authors investigated the existence and stability of such solutions for a sufficiently differentiable firing rate function $f$. They used Fourier techniques to reduce the problem of finding $\mathrm{N}$-bump solutions of Eq. (1) in the study of $\mathrm{N}$-bump solutions of an associated fourth-order ODE. For type (4) or type (6) coupling functions, this transformation is possible since their Fourier transforms possess a simple, rational polynomial structure [1]. For the case of the Heaviside function $f$, the authors conjectured that both stable and unstable $N$-bump solutions might exist. Following this conjecture, and considering the mathematical results for twobump solutions, the existence and stability of $\mathrm{N}$-bump solutions in a field with external inputs is studied analytically and numerically in Section 5. 
It should be stressed that great care has to be taken when comparing numerical solutions on a finite domain with solutions of the associated continuum neural field equation. It has been widely acknowledged that both boundary conditions and the spatial discretization of the numerical scheme may suggest stable solutions that do not exist in the continuum limit [24]. Using Matlab [25], we apply a Euler scheme with a sufficiently fine discretization mesh (typically $d x=0.005$ ) so as to integrate Eq. (1) to a steady state. The boundary points evolve freely according to the scheme, and the domain size is chosen so that the stationary solution is unaffected by the boundaries (see Appendix B for details).

\section{Analysis of one-bump solutions}

Following the approach presented in [14,26], we address in this section the existence and stability of one-bump solutions of Eq. (1) with external input $S_{1 b}(x)$ and a coupling function given by (6). We start with a theorem that establishes for a general class of coupling functions satisfying $\left(\mathrm{H}_{1}\right)$ and $\left(\mathrm{H}_{2}\right)$ necessary conditions for the existence.

Theorem 1. Suppose that hypotheses $\left(\mathrm{H}_{1}\right),\left(\mathrm{H}_{2}\right),\left(\mathrm{SH}_{1}\right)-\left(\mathrm{SH}_{3}\right)$ hold. The equation $u(x)=W\left(x+\frac{a}{2}\right)-W\left(x-\frac{a}{2}\right)-h+S_{1 b}(x)$ defines $a$ one-bump solution with $R[u]=\left(-\frac{a}{2}, \frac{a}{2}\right)$ if and only if the following three conditions are satisfied

(i) $W\left(x+\frac{a}{2}\right)-W\left(x-\frac{a}{2}\right)-h+S_{1 b}(x)=0$, for $x=\frac{a}{2}$,

(ii) $W\left(x+\frac{a}{2}\right)-W\left(x-\frac{a}{2}\right)-h+S_{1 b}(x)>0$, for $x \in\left[0, \frac{a}{2}\right)$,

(iii) $W\left(x+\frac{a}{2}\right)-W\left(x-\frac{a}{2}\right)-h+S_{1 b}(x)<0$, for $x>\frac{a}{2}$.

The proof of this theorem is given in Appendix A.

For lateral inhibition type connectivity and $S(x)=0 \forall x$, it follows that $u(x)=W\left(x+\frac{a}{2}\right)-W\left(x-\frac{a}{2}\right)-h$ defines a onebump solution if the condition $W(a)=h$ (which is equivalent to condition (i) of Theorem 1) is satisfied. However, for a coupling function $w(x)$ defined by (6) this is not true. Consider the examples $A=2, \alpha=0.3, k=0.08$ with $h=W(11.5)$ (Fig. 2, top left) and $A=2, \alpha=0.3, k=0.05$ with $h=W(9)$ (Fig. 2, top right). The solutions of $W(a)=h$ are $a=11.5$ and $a=9$, respectively, but $u(x)$ is not negative for all $x>a$ as can be seen in the bottom panels.

For a given $h>0$, if $\left(\mathrm{H}_{1}\right),\left(\mathrm{H}_{2}\right),\left(\mathrm{H}_{5}\right)$ and $\left(\mathrm{H}_{6}\right)$ hold, equation (i) of Theorem 1 may have zero, one or multiple solutions. Fig. 2 (top left) illustrates that there is no solution if $h>W\left(z_{1}\right)$, there is one solution if $h=W\left(z_{1}\right)$ or $h<W\left(z_{2}\right)$, there are exactly two positive solutions if $W\left(z_{3}\right)<h<W\left(z_{1}\right)$, and there are at least three solutions if $W\left(z_{2}\right)<h \leq W\left(z_{3}\right)$.

Let $a$ be a value that satisfies condition $W(a)=h$. From the two examples of Fig. 2 we conjecture that if $h<W\left(z_{3}\right)$ or $W\left(z_{2}\right)<$ $0, u(x)=W\left(x+\frac{a}{2}\right)-W\left(x-\frac{a}{2}\right)-h$ might not define a singlebump solution. In order to derive a sufficient condition for the existence for the case $S(x)=0 \forall x$, we add the following hypothesis on the coupling function of type (6):

$\left(\mathrm{H}_{7}\right) W\left(z_{2}\right)>0$.

Note that $W\left(z_{2}\right)$ is negative if the value of $k$ is sufficiently small, i.e., when the oscillations of $w(x)$ are more pronounced.

Theorem 2. Assume that hypotheses $\left(\mathrm{H}_{1}\right),\left(\mathrm{H}_{2}\right),\left(\mathrm{H}_{5}\right)-\left(\mathrm{H}_{7}\right)$ hold. If $W\left(z_{3}\right)<h<W\left(z_{1}\right)$ and $a \in\left(z_{1}, z_{2}\right)$ is solution of $W(a)=h$, then

$u(x)=W\left(x+\frac{a}{2}\right)-W\left(x-\frac{a}{2}\right)-h$

defines a stable one-bump solution with $R[u]=\left(-\frac{a}{2}, \frac{a}{2}\right)$.
The proof of this theorem is given in Appendix A.

In the following, we derive for the family of coupling functions with the integral given by (9) conditions on the parameters $\alpha$ and $k$ so that the conditions of Theorem 2 are satisfied. Suppose $W\left(z_{2}\right)>$ 0 , to conclude that $u(x)=W\left(x+\frac{a}{2}\right)-W\left(x-\frac{a}{2}\right)-h$ defines a single-bump solution, by Theorem 2, $W\left(z_{3}\right)<h<W\left(z_{1}\right)$ must hold. If we choose $h=W\left(\frac{\pi}{\alpha}\right)$, one solution of $h=W(a)$ is $a=\frac{\pi}{\alpha}$. Since $\frac{\pi}{\alpha} \in\left(z_{1}, z_{2}\right)$, the condition $h<W\left(z_{1}\right)$ holds. To show that $W\left(\frac{\pi}{\alpha}\right)^{\alpha}>W\left(z_{3}\right)$ also holds, consider the following two important properties of $(9)$.

Lemma 1. Let $n$ be a natural number, $x \geq 0$ and $W(x)$ defined by (9). Then

$$
\begin{aligned}
& W\left(x+\frac{n \pi}{\alpha}\right) \\
& =\left\{\begin{array}{ll}
-e^{-\frac{k n \pi}{\alpha}} W(x)+p_{1} p_{2}\left(1+e^{-\frac{k n \pi}{\alpha}}\right), & \text { if } n \text { is odd } \\
e^{-\frac{k n \pi}{\alpha}} W(x)+p_{1} p_{2}\left(1-e^{-\frac{k n \pi}{\alpha}}\right), & \text { if } n \text { is even }
\end{array},\right.
\end{aligned}
$$

and

$$
\begin{aligned}
& W\left(x-\frac{n \pi}{\alpha}\right) \\
& \quad=\left\{\begin{array}{ll}
-e^{\frac{k n \pi}{\alpha}} W(x)+p_{1} p_{2}\left(1+e^{\frac{k n \pi}{\alpha}}\right), & \text { if } n \text { is odd } \\
e^{\frac{k n \pi}{\alpha}} W(x)+p_{1} p_{2}\left(1-e^{\frac{k n \pi}{\alpha}}\right), & \text { if } n \text { is even }
\end{array},\right.
\end{aligned}
$$

hold, if $x-\frac{n \pi}{\alpha}>0$.

The proof of this lemma is given in Appendix A.

Lemma 2. Assume that $W(x)$ is defined by (9) and that $\left(\mathrm{H}_{7}\right)$ holds. Then $W\left(\frac{\pi}{\alpha}\right)>W\left(z_{3}\right)$.

The proof of this lemma is given in Appendix A.

In conclusion, for $h=W\left(\frac{\pi}{\alpha}\right)$, if we choose $\alpha=\frac{\pi}{a}$ and $k$ such that $W\left(z_{2}\right)>0$, the conditions (12) and (14) are satisfied. By Lemma 2, it follows that $h>W\left(z_{3}\right)$ holds. Consequently, by Theorem 2, $u(x)=W\left(x+\frac{a}{2}\right)-W\left(x-\frac{a}{2}\right)-h$ defines a stable one-bump solution.

For applications of the dynamic field model it is important to understand how the shape of the stationary bump changes as the parameters defining the spatial ranges of excitation and inhibition are changed [7]. For a rest state defined by $h=W\left(\frac{\pi}{\alpha}\right)$, Fig. 3 shows the bump width $a$ as a function of $\alpha$ (left) and $k$ (right) in the parameter ranges for which condition $\left(\mathrm{H}_{7}\right), W\left(z_{2}\right)>0$, is satisfied. It confirms Amari's result that a larger, stable bump (solid line) co-exists with a smaller, unstable bump (dashed line) [14]. Note that the two solution curves for the stable and unstable bumps do not meet since $h<W\left(z_{1}\right)$ holds (Theorem 2). Numerical simulations indicate that for $\alpha>0.433$ (or $k<0.076$ ) the single bump is destabilized to a periodic pattern (Fig. 4). This is consistent with findings in studies with a Mexican-hat coupling function, which describe the emergence of a global periodic pattern as the global inhibition parameter $h$ is reduced [14,21]. Note that $h=W\left(\frac{\pi}{0.44}\right)<W\left(\frac{\pi}{0.433}\right)$ holds for the example shown in Fig. 4.

It is important to notice that the width of the stable bump increases significantly when $\alpha$ is reduced whereas it is not affected by a change of $k$ over the tested range. It can be expected that this dependency of the excitation length on $\alpha$ also holds for solutions with multiple bumps. In Section 5.1, we discuss the existence of stable $N$-bump solutions (for $N>1$ ) in a given finite domain as a function of the parameter $\alpha$.

With the working memory application in mind, we discuss in the following the formation of a steady state excitation pattern when the stationary input $S_{1 b}(x)$ is applied for a sufficiently long 


\section{ARTICLE IN PRESS}
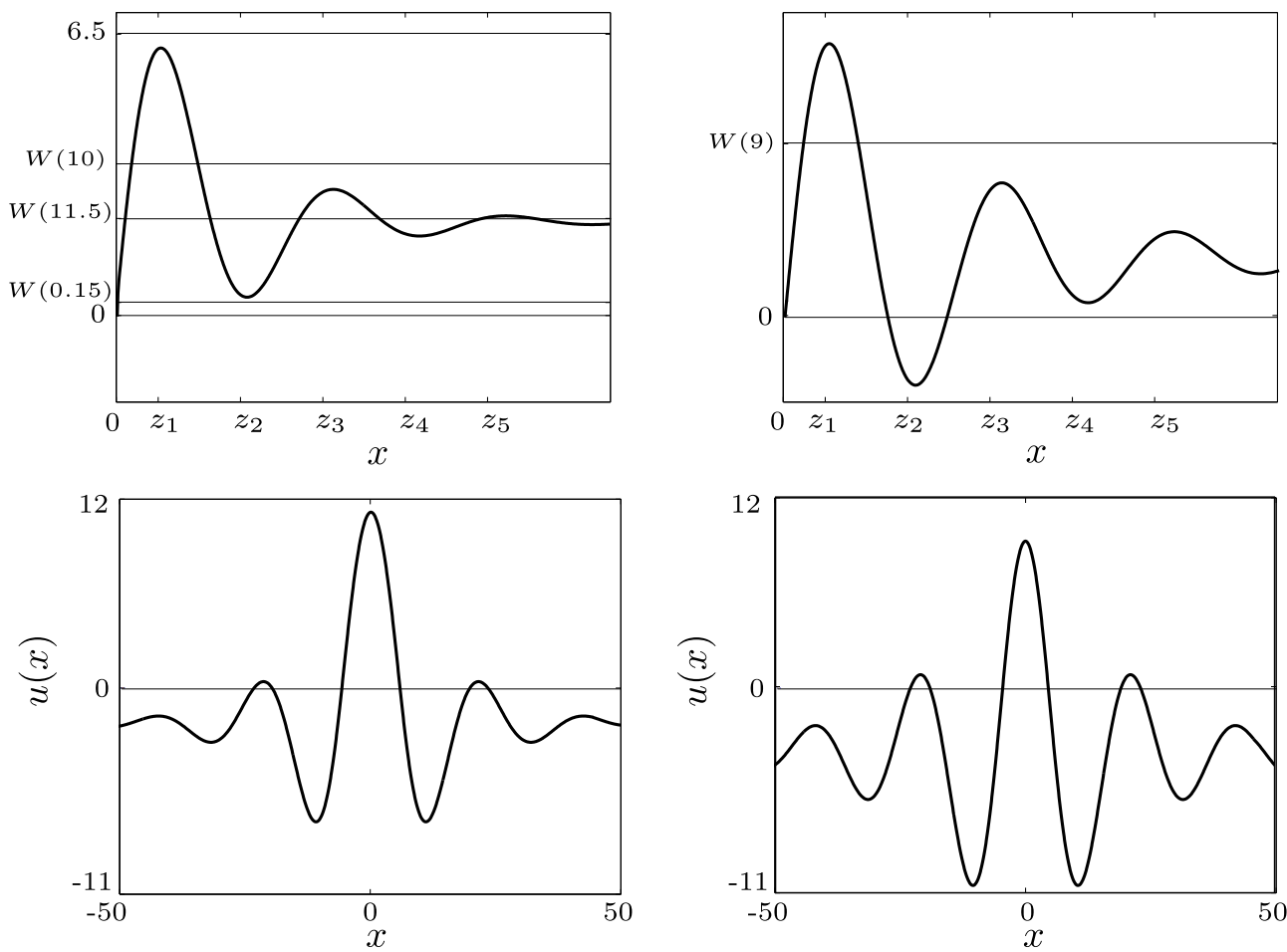

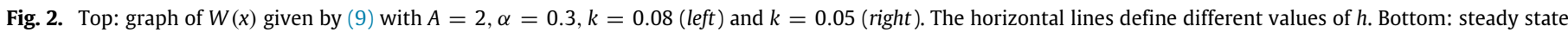
solution (11) for $S(x)=0$ and $W(x)$ given by (9) with $A=2, \alpha=0.3$ and $k=0.08$ (left) and $k=0.05$ (right). The values of $h$ are $W(11.5)$ (left) and $W(9)$ (right).
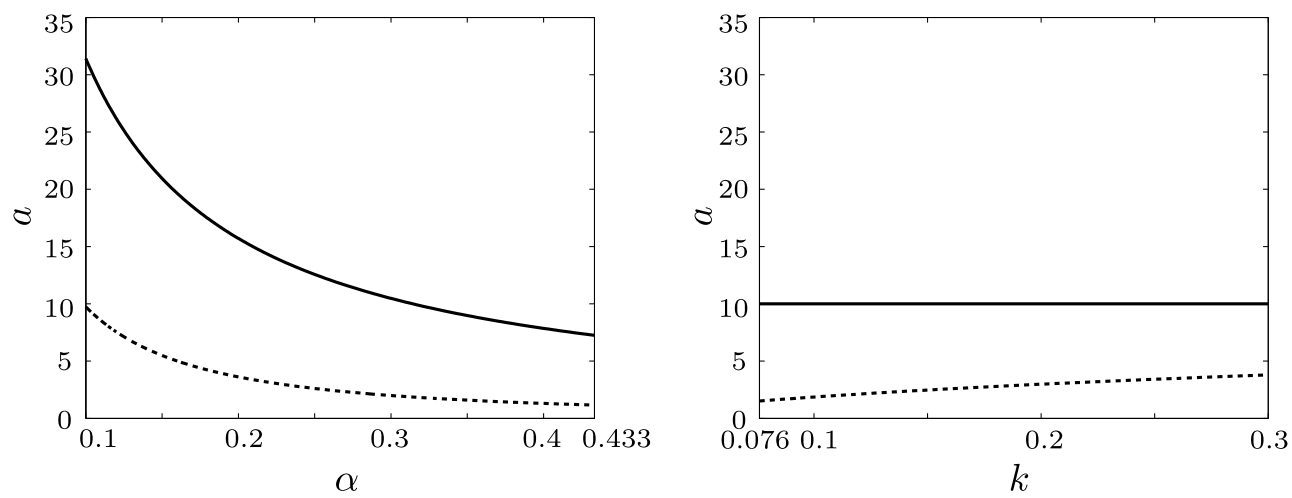

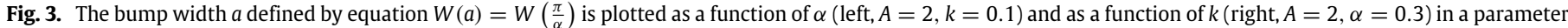
range for which condition $W\left(z_{2}\right)>0$ is satisfied. The solid lines represent stable $\left(a \in\left(z_{1}, z_{2}\right)\right)$ and the dashed lines unstable $\left(a \in\left(0, z_{1}\right)\right)$ bump solutions.
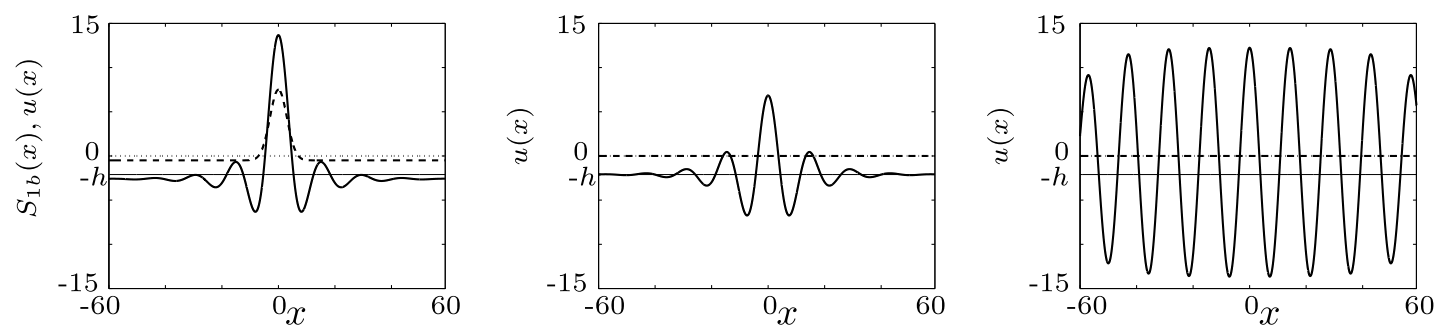

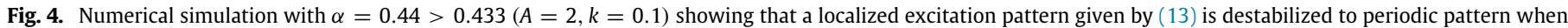

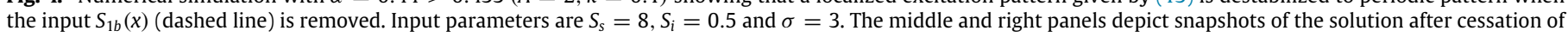
the input, at times $t=75$ and $t=200$, respectively.

time interval $[0, T]$. This pattern should converge to a stable onebump solution after cessation of the input, $S_{1 b}(x)=0 \forall x$, at time $t=T$.

If the initial state of the field is $u(x)=-h<0 \forall x$, two situations may occur depending on the input strength [27]:
- the field remains in an input-driven state if $S_{1 b}(0) \leq h$, that is, $u(x)=S_{1 b}(x)-h$;

- the field develops one or more localized bumps if $S_{1 b}(0)>h$.

The excited region created by a sufficiently strong external input depends on the input shape as illustrated in Fig. 5. The 

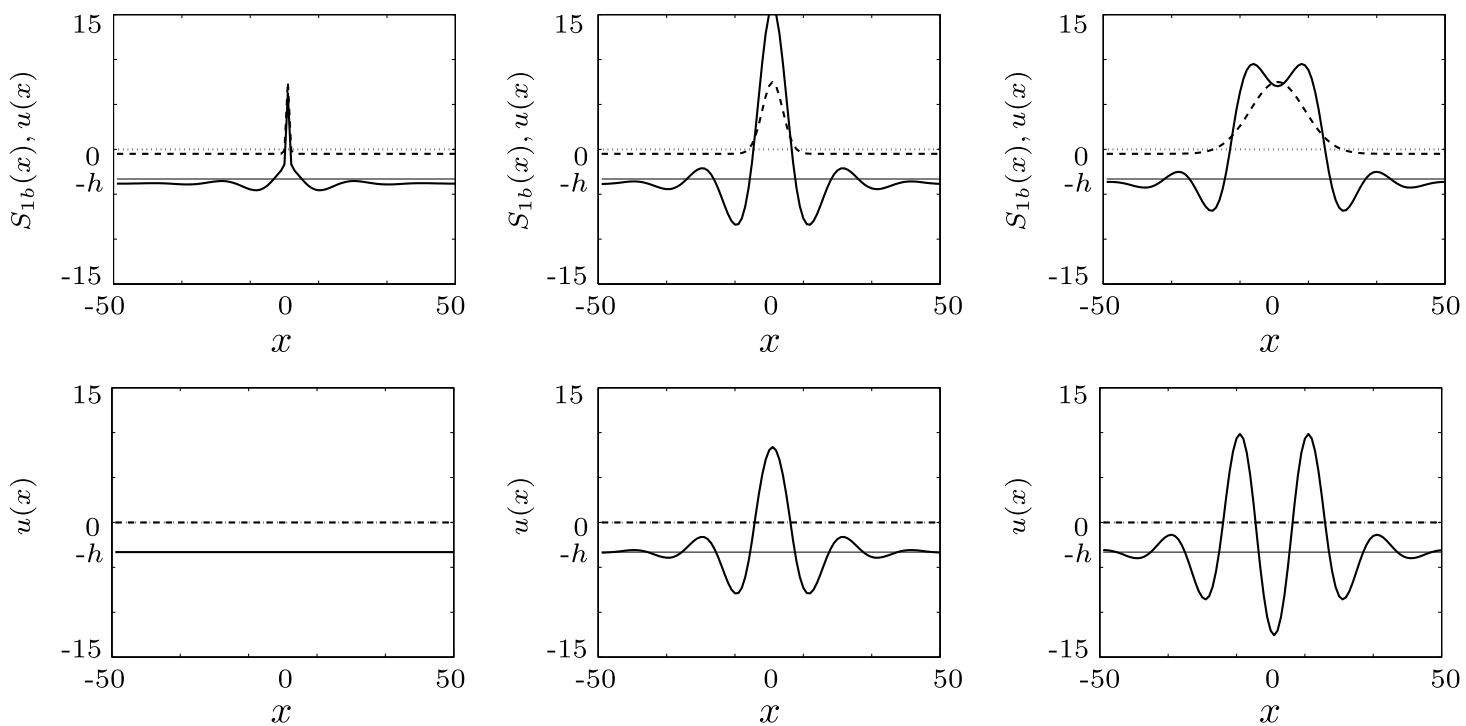

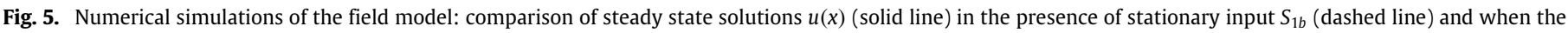

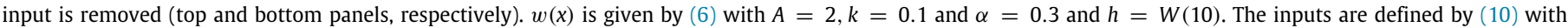
$S_{s}=8, S_{i}=0.5$ and different standard deviations, $\sigma=0.4$ (left), $\sigma=3$ (middle), $\sigma=8$ (right).

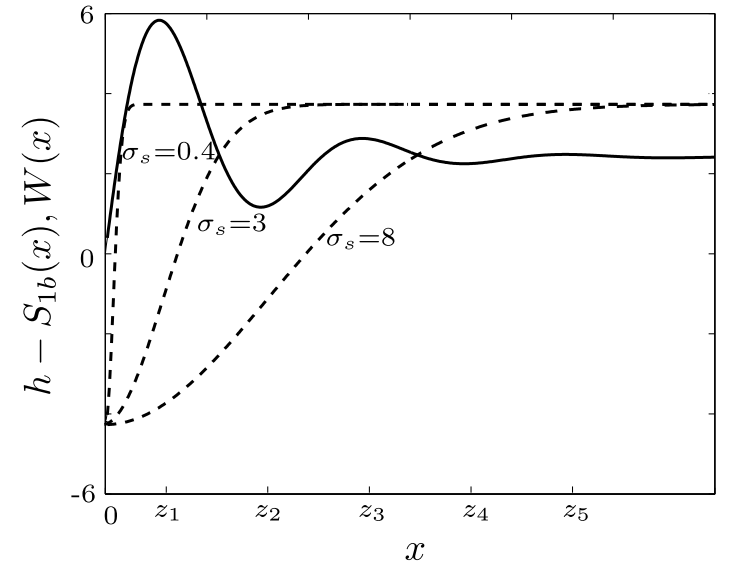

Fig. 6. An example of the intersection of $h-S_{1 b}\left(\frac{x}{2}\right)$ (dashed line) with $W(x)$ (solid line) defined by (9) with $A=2, \alpha=0.3$ and $k=0.1$, and $h=W(10)$. The input parameters are $S_{s}=8, S_{i}=0.5$, and $\sigma_{s} \in\{0.4,3,8\}$.

numerical simulations of the model show that inputs given by (10) of equal amplitude but three different widths all trigger the evolution of a localized excitation (top). However, only the pattern of intermediate width converges to a stable one-bump when the input is removed at time $t=T$ (Fig. 5, middle). A very narrow input is not able to destabilize the homogeneous resting state $u(x)=-h \forall x$ (the width of the initial excitation is smaller than the width of the unstable bump), whereas a pattern triggered by a broad input converges to a stable two-bump solution.

Fig. 6 compares the graphs of the solutions of Eq. (12) for the three cases. The intersections of $h-S_{1 b}\left(\frac{x}{2}\right)$ with $W(x)$ illustrate the sufficient conditions on the input shape, which guarantee the existence of a unique solution of Eq. (12).

Theorem 3. Assume that hypotheses $\left(\mathrm{H}_{1}\right),\left(\mathrm{H}_{2}\right),\left(\mathrm{H}_{5}\right)-\left(\mathrm{H}_{7}\right)$, and $\left(\mathrm{SH}_{1}\right)-\left(\mathrm{SH}_{3}\right)$ hold. If $W\left(z_{3}\right)<h<W\left(z_{1}\right), S_{1 b}(0)>h, S_{1 b}\left(\frac{z_{1}}{2}\right)>$ 0 and $S_{1 b}\left(\frac{z_{2}}{2}\right)<0$, then the equation

$h-S_{1 b}\left(\frac{x}{2}\right)=W(x)$

has a unique positive solution a that belongs to the interval $\left(z_{1}, z_{2}\right)$.

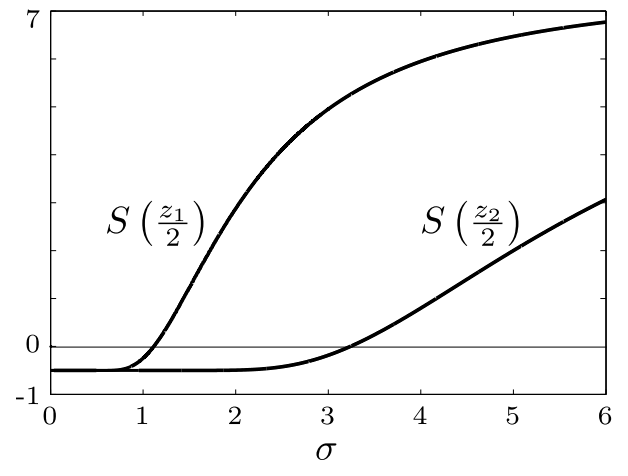

Fig. 7. Graphs of $S_{1 b}\left(\frac{z_{1}}{2}\right)$ and $S_{1 b}\left(\frac{z_{2}}{2}\right)$ as a function of $\sigma \in[0,6]$.

The proof of this theorem is given in Appendix A.

Note that since $w(x)<0$ for $z_{1}<x<z_{2}$, and $S^{\prime}\left(\frac{x}{2}\right)<0$ for all $x>0$ hold, the equilibrium local excitation of width $a$ satisfies the stability condition (14).

The following numerical example illustrates the range of input widths that lead to a stable one-bump solution. Consider the coupling function $w(x)$ given by (6) with $A=2, k=0.1$, and $\alpha=\frac{\pi}{10}, h=W(10)$, and $S_{1 b}(x)$ given by (10) with $S_{s}=8, S_{i}=0.5$ and $\sigma>0$. In this example, $S_{1 b}(0)=7.5>W(10)$, thus by Theorem 3, if $S\left(\frac{z_{1}}{2}\right)>0$ and $S\left(\frac{z_{2}}{2}\right)<0$ there exists a value $a \in\left(z_{1}, z_{2}\right)$ such that $W(10)-S\left(\frac{a}{2}\right)=W(a)$. Fig. 7 shows the values of $S_{1 b}\left(\frac{z_{1}}{2}\right)$ and $S_{1 b}\left(\frac{z_{2}}{2}\right)$ as a function of $\sigma \in[0,6]$. Since $S_{1 b}\left(\frac{z_{1}}{2}\right)>0$ at $\sigma>1.1290$ and $S_{1 b}\left(\frac{z_{2}}{2}\right)<0$ at $\sigma<3.2523$, we can conclude that for $1.1290<\sigma<3.2523$ there exists a value $a \in\left(z_{1}, z_{2}\right)$ such that $W(10)-S\left(\frac{a}{2}\right)=W(a)$.

It is easy to see that the steady state excitation pattern generated by the input is in the basis of attraction of the equilibrium width solution $a=\frac{\pi}{\alpha}$ when the input is removed. Let $a(t)$ be the width of the excited region at time $t$. For the case $S_{1 b}(x)=0$ for all $x$, the equation describing the change of $a(t)$ as $t \rightarrow \infty$ is given by

$\frac{d a}{d t}=\frac{1}{c}[W(a)-h]$

with $c=-\frac{\partial u(a)}{\partial x}>0$. 


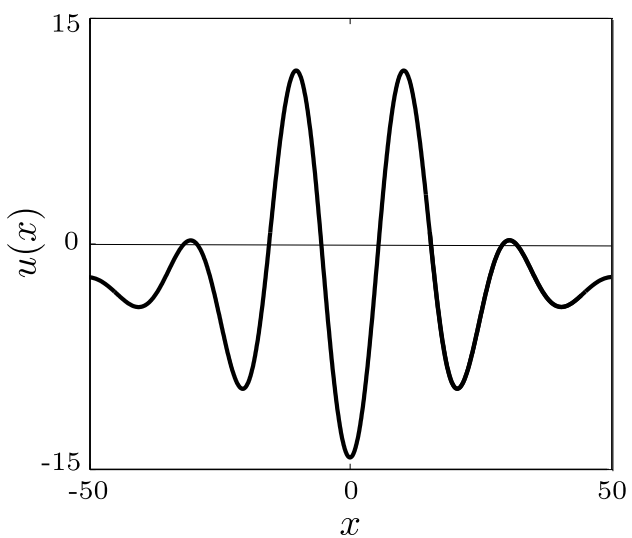

Fig. 8. Steady state solution $u(x)$ defined by (16) for $W(x)$ given by (9) with $A=2, \alpha=\frac{\pi}{10}, k=0.08$, and $h=W(10), a=10, b=-\frac{\arctan \left(\frac{10 \pi+100}{8-125 \pi}\right)}{\alpha}+20$.

The width increases if $\frac{d a}{d t}>0$, decreases if $\frac{d a}{d t}<0$ and does not change if $\frac{d a}{d t}=0$. Let $h=W\left(\frac{\pi}{\alpha}\right)$ and $W\left(z_{2}\right)>0$, the width increases if $a<\frac{\pi}{\alpha}$, decreases if $a>\frac{\pi}{\alpha}$ and does not change if $a=\frac{\pi}{\alpha}$. In all cases, the width will tend to the equilibrium width $a=\frac{\pi}{\alpha}$.

\section{Analysis of two-bump solutions}

In this section, we study the existence and stability of twobump solutions in the presence of a transient input inhomogeneity $S_{2 b}(x)$. We start by proving for the case $S_{2 b}(x)=0 \forall x$ the existence of a $a$-quasi-solution. We then use a numerical example to show that the conditions established in Theorem 3 for a single-bump solution are not sufficient to guarantee the correct positive ranges of a stable two-bump solution.

Theorem 4. Assume that the coupling function $w(x)$ is of type (6), and $\tau$ is a triple of the form $(a, b, a+b)$, then there exists a a-quasisolution $u_{\tau}$ of (1).

The proof of this theorem is given in Appendix A.

Two-bump solutions are stable if conditions (19) are satisfied. Considering the zeros $z_{n}$ of $w(x)$ defined by (6), if $b \in\left(z_{2 n}, z_{2 n+1}\right)$ for some $n \in \mathbb{N}$, then

$w(b)>0, \quad w\left(b-\frac{\pi}{\alpha}\right)<0, \quad w\left(b+\frac{\pi}{\alpha}\right)<0$.

Thus, if $a=\frac{\pi}{\alpha}$ and $b \in\left(z_{2 n}, z_{2 n+1}\right)$ for some $n \in \mathbb{N}$, the solution is stable. Consider $h=W\left(\frac{\pi}{\alpha}\right)$, by Theorem 4 there exists a triple $(a, b, c)$, with $a=\frac{\pi}{\alpha}, b>a$ (that is, $\left.W(b)=p_{1} p_{2}\right)$ and $c=b+\frac{\pi}{\alpha}$, that satisfies the conditions (17) and (18) with $S(x)=0 \forall x$. If $b \in\left(z_{2}, z_{3}\right)$, this solution is stable.

Now consider as a specific example $A=2, \alpha=\frac{\pi}{10}, k=0.08$. For these values, $\left(\mathrm{H}_{1}\right),\left(\mathrm{H}_{2}\right),\left(\mathrm{H}_{6}\right)$ and $\left(\mathrm{H}_{7}\right)$ are satisfied. Assuming $h=W(10)$, by Lemma 2 , and as a consequence of Theorem 2, it follows that $u(x)=W(x)+W(x-10)-h$ defines a single-bump solution. On the other hand, by Theorem 4 , if $a=10$ and $b=$ $-\frac{\arctan \left(\frac{10 \pi+100}{8-125 \pi}\right)}{\alpha}+20 \in\left(z_{2}, z_{3}\right)$, we have $u_{\tau}(0)=u_{\tau}(a)=u_{\tau}(b)=$ $u_{\tau}(a+\stackrel{\alpha}{b})=0$ which is equivalent to $u\left(-\frac{a+b}{2}\right)=u\left(-\frac{b-a}{2}\right)=$ $u\left(\frac{b-a}{2}\right)=u\left(\frac{a+b}{2}\right)=0$. However, Fig. 8 shows that $u(x)=$ $W\left(x+\frac{b+a}{2}\right)-W\left(x+\frac{b-a}{2}\right)+W\left(x-\frac{b-a}{2}\right)-W\left(x-\frac{b+a}{2}\right)-W\left(\frac{\pi}{\alpha}\right)$ defines a solution with more than two regions of excitation.

In order to ensure the existence and the stability of a two-bump solution, we replace $\left(\mathrm{H}_{7}\right)$ by the following hypothesis:

$\left(\mathrm{H}_{8}\right) W\left(z_{2}\right)>\frac{p_{1} p_{2}}{\left(1+e^{\frac{2 k \pi}{\alpha}}\right)}$, with $p_{1}=\frac{A}{k^{2}+\alpha^{2}}, p_{2}=\alpha k+k$.
Theorem 5. Assume that for a coupling function $w(x)$ of type (6) the hypothesis $\left(\mathrm{H}_{8}\right)$ holds. If $a=\frac{\pi}{\alpha}$ and $b \in\left(z_{2}, z_{3}\right)$ such that $W(b)=$ $p_{1} p_{2}$, then

$$
\begin{aligned}
u(x)= & W\left(x+\frac{b+a}{2}\right)-W\left(x+\frac{b-a}{2}\right) \\
& +W\left(x-\frac{b-a}{2}\right)-W\left(x-\frac{b+a}{2}\right)-W\left(\frac{\pi}{\alpha}\right)
\end{aligned}
$$

defines a stable two-bump solution with $R[u]=\left(-\frac{b+a}{2},-\frac{b-a}{2}\right) \cup$ $\left(\frac{b-a}{2}, \frac{b+a}{2}\right)$.

The proof of this theorem is given in Appendix A.

In order to generalize the results obtained for a single localized input $S_{1 b}(x)$, we consider without restriction a symmetric, bimodal input centered at $x=0$ and defined by

$S_{2 b}(x)=S_{1 b}\left(x-x_{c}\right)+S_{1 b}\left(x+x_{c}\right)+S_{i}$

where $x_{c}$ is a positive constant. Since the positive input range of $S_{1 b}(x)$ is $\left(\overline{x_{1}}, \overline{x_{2}}\right)$, the distance between input bumps is defined by $d_{s}=2 \overline{x_{1}}$.

When the field is initially at resting state $u(x)=-h<$ $0 \forall x$, a sufficiently strong input, $S\left(\frac{\overline{x_{2}}-\overline{x_{1}}}{2}\right)>h$, may trigger the evolution of different patterns of excitation depending on the value $x_{c}$. This is shown in the numerical simulation of Fig. 9 (top) where three different distances $x_{c}$ were tested for an otherwise identical bimodal input. For a small distance, the field evolves a single region of excitation, whereas for larger distances a two-bump solution emerges. Like in the discussion of the input-induced one-bump, it is important to understand what happens when at time $t=T$ the excitation pattern has reached a steady state and the bimodal input is removed (Fig. 9, bottom). In fact, for the largest distance tested, the lateral excitation from the existing bumps proves to be sufficient to create a third bump at an intermediate position, whereas for the smallest distance, the pattern converges to a stable one-bump solution (for a discussion of the pattern formation process see Section 5.1). Implementing a precise working memory function thus requires to determine constraints on the input shape, which guarantee the existence and stability of an input-induced two-bump solution like the example depicted in the middle panels.

Suppose that $h=W\left(\frac{\pi}{\alpha}\right)$, and that hypothesis $\left(\mathrm{H}_{8}\right)$ holds. By Theorem $5, u(x)=W(x)-W(x-a)+W(x-b)-W(x-a-b)-h$, with $a=\frac{\pi}{\alpha}$ and $b \in\left(z_{2}, z_{3}\right)$ such that $W(b)=p_{1} p_{2}$, defines a stable two-bump solution. We know that if $a \in\left(z_{1}, z_{2}\right), b \in$ $\left(z_{2}, z_{3}\right)$ and $a+b \in\left(z_{3}, z_{4}\right)$ the conditions for stability (19) are satisfied. Thus, we introduce the subset $\Omega$ of $\mathbb{R}^{2}$ (see Fig. 10) defined as

$$
\begin{aligned}
\Omega= & \left\{(x, y) \in \mathbb{R}^{2} \mid x>z_{1} \wedge x\right. \\
& \left.+z_{1}<y<x+z_{2} \wedge-x+z_{3}<y<-x+z_{4}\right\} .
\end{aligned}
$$

The following theorem gives sufficient conditions for the existence of values $(a, b) \in \Omega$ that satisfy the conditions (17) and (18) if $h=W\left(\frac{\pi}{\alpha}\right)$.

Theorem 6. Assume that the coupling function $w(x)$ is given by (6), and that hypotheses $\left(\mathrm{H}_{8}\right),\left(\mathrm{SH}_{1}\right),\left(\mathrm{SH}_{4}\right)$ and $\left(\mathrm{SH}_{5}\right)$ hold. If $S_{2 b}\left(\frac{\overline{x_{2}}-\overline{x_{1}}}{2}\right)>W\left(\frac{\pi}{\alpha}\right), S_{2 b}\left(\frac{z_{1}}{2}\right)<0, S_{2 b}\left(\frac{z_{2}}{2}\right)>0, S_{2 b}\left(\frac{z_{3}}{2}\right)>0$ and $S_{2 b}\left(\frac{z_{4}}{2}\right)<0$, then there exists a point $(a, b)$ belonging to the region $\Omega \subset \mathbb{R}^{2}$ (30) such that

$W(a)+W(b)-W(b-a)-W\left(\frac{\pi}{\alpha}\right)+S_{2 b}\left(\frac{b-a}{2}\right)=0$, 

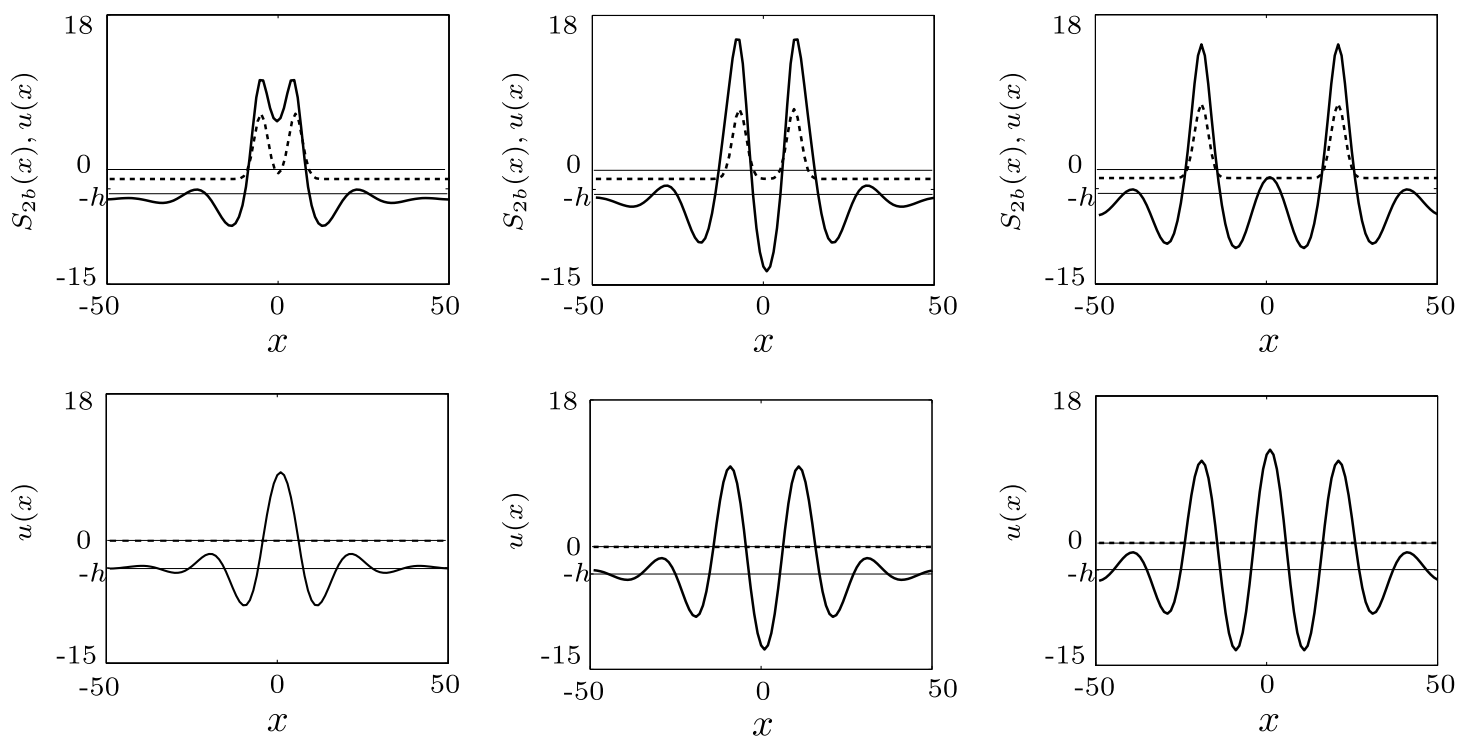

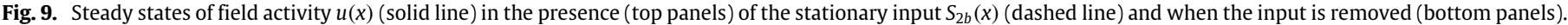

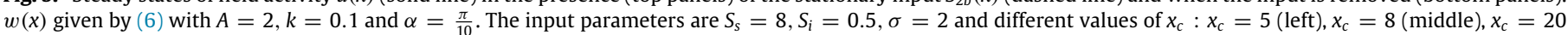
(right).

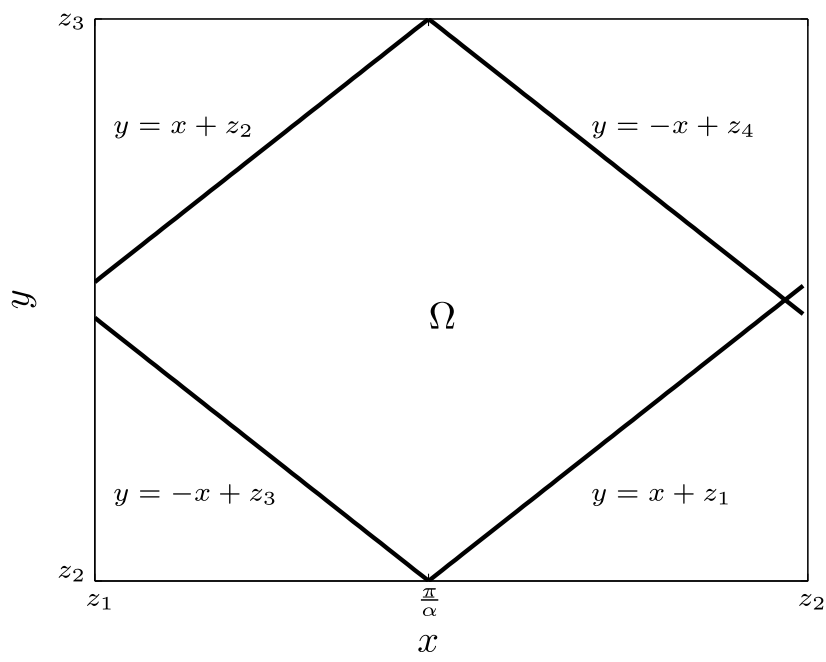

Fig. 10. Region $\Omega \subset \mathbb{R}^{2}$ defined by (30).

and

$W(a)-W(b)+W(a+b)-W\left(\frac{\pi}{\alpha}\right)+S_{2 b}\left(\frac{b+a}{2}\right)=0$.

The proof of this theorem is given in Appendix A.

We illustrate Theorem 6 with an example. Consider the coupling function $w(x)$ of type (6) with $A=2, k=0.1$, and $\alpha=$ $\frac{\pi}{10}, h=W(10)$, and the input $S_{2 b}(x)$ defined by (29) with $S_{s}=$ 6, $S_{i}=1, \sigma=\frac{\pi}{2 \alpha \sqrt{\ln \left(S_{s}^{2}\right)-\ln \left(S_{i}^{2}\right)}}$ such that the width of each positive region is equal to $\frac{\pi}{\alpha}$. In this example we have $S_{2 b}\left(x_{c}\right)=5>W(10)$. By Theorem 6, if $S_{2 b}\left(\frac{z_{1}}{2}\right)<0, S_{2 b}\left(\frac{z_{2}}{2}\right)>0, S_{2 b}\left(\frac{z_{3}}{2}\right)>0$ and $S_{2 b}\left(\frac{z_{4}}{2}\right)<0$, then there exists a point $(a, b)$ belonging to $\Omega$ given by (30) such that Eqs. (31) and (32) are satisfied. In this example, $x_{c}$ should be larger than $\frac{z_{1}}{2}+\frac{\pi}{2 \alpha}=\frac{z_{2}}{2}$ such that condition $S_{2 b}\left(\frac{z_{1}}{2}\right)<0$ is fulfilled, and, on the other hand, $x_{c}$ should be smaller than $\frac{z_{4}}{2}-$ $\frac{\pi}{2 \alpha}=\frac{z_{3}}{2}$ such that condition $S_{2 b}\left(\frac{z_{4}}{2}\right)<0$ is fulfilled. As the width of each bump of $S_{2 b}$ is $\frac{\pi}{\alpha}$, and $\frac{z_{2}}{2}<x_{c}<\frac{z_{3}}{2}$, we have that $S_{2 b}\left(\frac{z_{2}}{2}\right)>0$ and that $S_{2 b}\left(\frac{z_{3}}{2}\right)<0$ because $x_{c}-\frac{\pi}{2 \alpha}<\frac{z_{2}}{2}$ and $x_{c}+\frac{\pi}{2 \alpha}>\frac{z_{3}}{2}$.
As $h=W\left(\frac{\pi}{\alpha}\right)$, by Theorem 4 , there exists a triple $\left(a_{1}, b_{1}, c_{1}\right)$ with $a_{1}=\frac{\pi}{\alpha}, b_{1}=-\frac{\arctan \left(\frac{p_{2}}{p_{3}}\right)}{\alpha}+\frac{2 \pi}{\alpha}$ and $c_{1}=a_{1}+b_{1}$, which satisfies conditions (17) and (18) for the case $S(x)=0$ for all $x$. Fig. 11 shows numerical simulations for two different values of $x_{c}$, one for which the distance between the input bumps $d_{s}$ is less than $b_{1}-a_{1}$, and another for which this distance is larger than $b_{1}-a_{1}$. In both cases, the initial two-bump solution in the presence of the bimodal input $S_{2 b}(x)$ (top) converges to the solution defined by the triple $\left(a_{1}, b_{1}, c_{1}\right)$ when the input is removed (bottom). The panels on the right illustrate this result by showing the curves in $\Omega$ implicitly defined by Eqs. (31) (dashed-dotted line) and (32) (dotted line), respectively. Their point of intersection represents the solution. For the two examples, the shape of the input-induced patterns is relatively close to the shape of the equilibrium solution $\left(\frac{\pi}{\alpha}, b_{1}\right)$ without input. To show that the equilibrium is indeed asymptotically stable, that is, any trajectory starting in $\Omega$ converges to $\left(\frac{\pi}{\alpha}, b_{1}\right)$ as $t$ increases, we perform a phase plane analysis of the dynamical system describing the motions of $a(t)$ and $b(t)$ after cessation of $S_{2 b}(x)$.

The planar system is given by

$$
\begin{aligned}
\frac{d a}{d t}= & \frac{1}{c_{1}}[W(a)-W(b)+W(a+b)-h] \\
& +\frac{1}{c_{2}}[W(a)+W(b)-W(b-a)-h], \\
\frac{d b}{d t}= & \frac{1}{c_{1}}[W(a)-W(b)+W(a+b)-h] \\
& +\frac{1}{c_{2}}[W(b-a)-W(a)-W(b)+h],
\end{aligned}
$$

where $c_{1}=\frac{\partial u(0, t)}{\partial x}$ and $c_{2}=-\frac{\partial u(a, t)}{\partial x}$.

The stationary solutions of (33) and (34) are given by

$W(a)+W(a+b)-W(b)-h=0$,

$W(a)+W(b)-W(b-a)-h=0$.

For $h=W\left(\frac{\pi}{\alpha}\right)$, we have proven in Theorem 4 that there exists a solution with $a_{1}=\frac{\pi}{\alpha}$ and $b_{1}=-\frac{\arctan \frac{p_{2}}{p_{3}}}{\alpha}+\frac{2 \pi}{\alpha}$ that satisfies conditions (35) and (36).

We define $G$ and $H$ to be the right hand sides of (33) and (34), respectively. To determine the type of the phase plane singularity, 


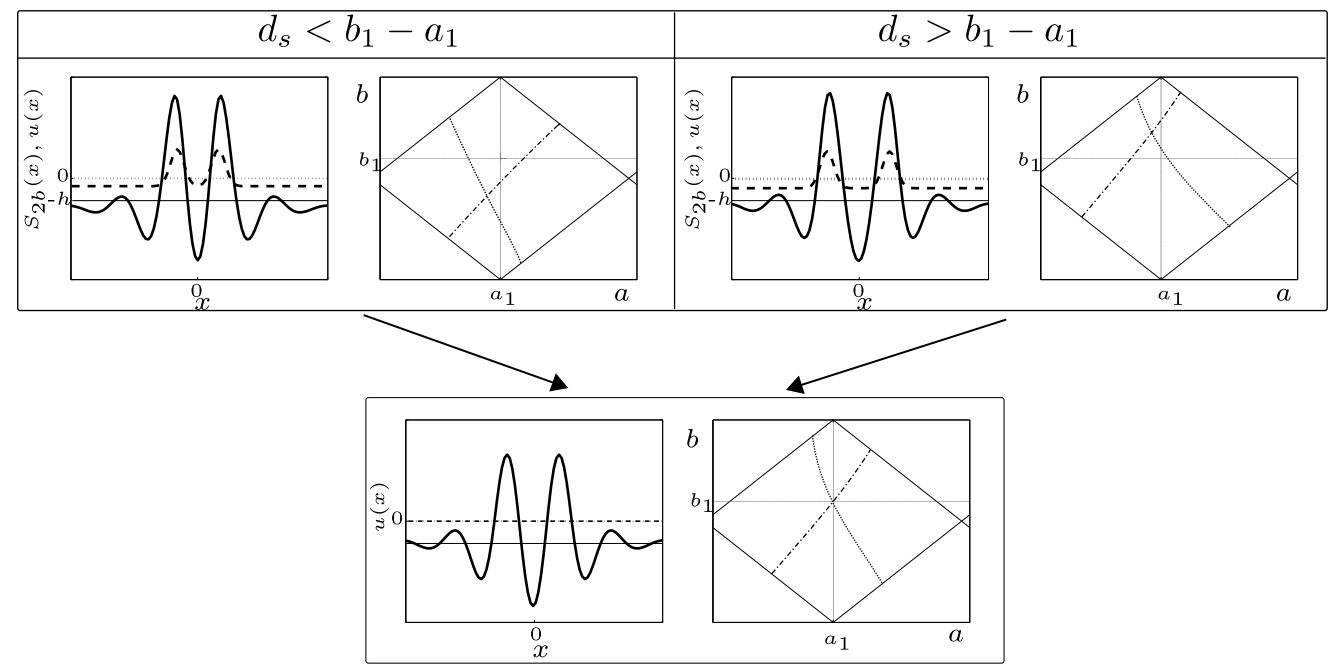

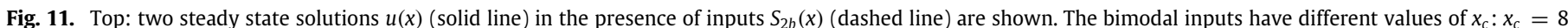

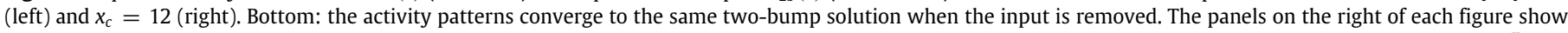

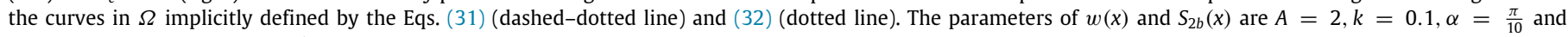
$S_{s}=6, S_{i}=1, \sigma=5(\ln (36))^{-\frac{1}{2}}$, respectively.

we linearize (33)-(34) around the equilibrium solution $(a, b)=$ $\left(a_{1}, b_{1}\right)$. The Jacobian matrix $J$ of the system at $\left(a_{1}, b_{1}\right)$ is given by

$J=\left(\begin{array}{ll}G_{a} & G_{b} \\ H_{a} & H_{b}\end{array}\right)$,

where $G_{a}, G_{b}, H_{a}$ and $H_{b}$ are partial derivatives evaluated at $\left(a_{1}, b_{1}\right)$. They are given by

$G_{a}=\frac{1}{c_{1}}\left[w\left(a_{1}\right)+w\left(a_{1}+b_{1}\right)\right]+\frac{1}{c_{2}}\left[w\left(a_{1}\right)+w\left(b_{1}-a_{1}\right)\right]$,

$G_{b}=\frac{1}{c_{1}}\left[w\left(a_{1}+b_{1}\right)-w\left(b_{1}\right)\right]+\frac{1}{c_{2}}\left[w\left(b_{1}\right)-w\left(b_{1}-a_{1}\right)\right]$,

$H_{a}=\frac{1}{c_{1}}\left[w\left(a_{1}\right)+w\left(a_{1}+b_{1}\right)\right]-\frac{1}{c_{2}}\left[w\left(a_{1}\right)+w\left(b_{1}-a_{1}\right)\right]$,

$H_{b}=\frac{1}{c_{1}}\left[w\left(b_{1}+a_{1}\right)-w\left(b_{1}\right)\right]+\frac{1}{c_{2}}\left[w\left(b_{1}-a_{1}\right)-w\left(b_{1}\right)\right]$.

The eigenvalues, $\lambda$, of $J$ satisfy

$\lambda^{2}-\left(G_{a}+H_{b}\right) \lambda+G_{a} H_{b}-G_{b} H_{a}=0$.

If the trace of $J$ is negative and the determinant of $J$ is positive, we can conclude that both eigenvalues of the Jacobian matrix have negative real parts.

$G_{a}+H_{b}<0$

and

$G_{a} H_{b}-G_{b} H_{a}>0$.

The behavior of solutions near $\left(a_{1}, b_{1}\right)$ is then qualitatively captured by the phase portrait of a stable node or a stable spiral. We have,

$$
\begin{aligned}
G_{a}+H_{b}= & {\left[\frac{1}{c_{1}}+\frac{1}{c_{2}}\right]\left[w\left(a_{1}\right)-w\left(b_{1}\right)\right] } \\
& +\frac{2}{c_{1}} w\left(b_{1}+a_{1}\right)+\frac{2}{c_{2}} w\left(b_{1}-a_{1}\right),
\end{aligned}
$$

and

$$
\begin{aligned}
G_{a} H_{b}-G_{b} H_{a} \\
=\frac{2}{c_{1} c_{2}}\left[w\left(a_{1}\right)-w\left(b_{1}\right)\right]\left[w\left(b_{1}-a_{1}\right)+w\left(a_{1}+b_{1}\right)\right] \\
\quad+\frac{4}{c_{1} c_{2}}\left[w\left(a_{1}+b_{1}\right) w\left(b_{1}-a_{1}\right)-w\left(a_{1}\right) w\left(b_{1}\right)\right] .
\end{aligned}
$$

For $a_{1}=\frac{\pi}{\alpha}$ and $b_{1}=-\frac{\arctan \frac{p_{2}}{p_{3}}}{\alpha}+\frac{2 \pi}{\alpha}$ it follows that $w\left(a_{1}\right)<0, \quad w\left(b_{1}\right)>0$, $w\left(b_{1}-a_{1}\right)<0, \quad w\left(b_{1}+a_{1}\right)<0$.

We can thus conclude that (43) and (44) are satisfied.

For the example shown in Fig. 11, Fig. 12 depicts trajectories of the system (33)-(34) with initial conditions $(a, b) \in \Omega$. The trajectories were generated using the "pplane8.m" tool [28]. For the general case, it is difficult to determine the direction of motion for arbitrary $(a, b) \in \Omega$. However, the linearization of system (33)-(34) suggests that in a local neighborhood all trajectories converge to the equilibrium solution $\left(a_{1}, b_{1}\right)$.

\section{Analysis of multi-bump solutions}

In this section, we address the formation of multi-bump solutions as a computational mechanism supporting a multi-item memory of external inputs. Our strategy is to follow the approach for the two-bump case and first derive necessary conditions for the existence and linear stability of multi-bumps in a field without input. We then report numerical evidence showing that the sufficient conditions for the input width and distance in terms of the coupling function, which we have derived in the previous section for a two-bump solution, can be exploited to implement a precise multi-item memory.

Recall that an $N$-bump solution is one whose region of excitation is the union of $N$ disjoint, finite and open intervals, that is, $R(u)=\left(a_{0}, a_{1}\right) \cup\left(a_{2}, a_{3}\right) \cup \cdots \cup\left(a_{2 N-2}, a_{2 N-1}\right)$. With the use of the Heaviside function (3), it follows from (2) and (21) that the steady state solution $u_{N}(x)$ can be written as

$u(x)=\sum_{i=0}^{N-1}\left(\int_{a_{2 i}}^{a_{2 i+1}} w(x-y) d y\right)-h$.

Using (7), a potential $N$-bump solution of Eq. (1) is then given by

$u(x)=\sum_{i=0}^{N-1}\left(W\left(x-a_{2 i}\right)-W\left(x-a_{2 i+1}\right)\right)-h$.

In generalization of the two-bump case, we consider only symmetric $N$-bump solutions $u(x)$ that are supported by the 
Table 1

Solutions of (50) for $N \in\{2,3,4,5,6\}$ when the coupling function $w(x)$ is defined by $A=2, \alpha=\frac{\pi}{10}$ and $k=0.1$.

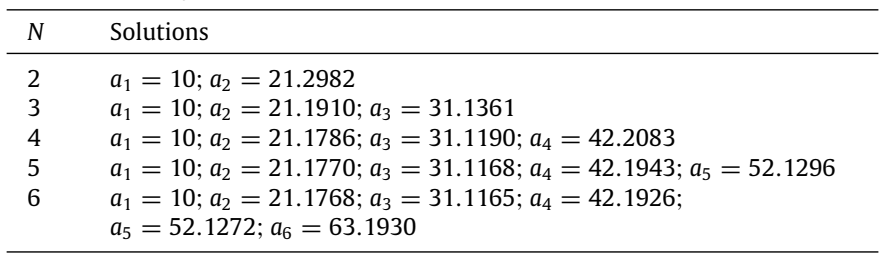

coupling function of type (6). Note that the symmetry of an $\mathrm{N}$ bump pattern does not necessarily imply equal width of all bumps and equal distance between individual bumps (see the definition of an $N$-bump equal width and distance solution in [23]). The following proposition states that for the symmetric case, the $2 \mathrm{~N}$ equations $u\left(a_{i}\right)=0$, which an $N$-bump solution must satisfy, can be reduced to a system of $N$ equations.

Proposition 1. Suppose that hypotheses $\left(\mathrm{H}_{1}\right),\left(\mathrm{H}_{2}\right),\left(\mathrm{H}_{5}\right)$ and $\left(\mathrm{H}_{6}\right)$ hold. If $u(x)$ is symmetric with respect to the point $\frac{a_{0}+a_{2 N-1}}{2}$, the system of equations

$\left\{\begin{array}{l}u\left(a_{0}\right)=0 \\ u\left(a_{1}\right)=0 \\ \cdots \\ u\left(a_{2 N-1}\right)=0\end{array}\right.$

can be reduced to

$\left\{\begin{array}{l}u\left(a_{0}\right)=0 \\ \cdots \\ u\left(a_{N}\right)=0 .\end{array}\right.$

The proof of this proposition is presented in Appendix A.

In the following examples, we assume without loss of generality that $a_{0}=0$. We apply Newton's method to approximate a solution of the nonlinear system (50). To discuss the stability of a stationary $\mathrm{N}$-bump solution, we follow the approach for the two-bump solution in the previous section and consider small perturbations of the steady state $u_{N}(x)$. The system of equations of motion for $a_{i}(t), i=1, \ldots, N$ can be linearized about the stationary solution. The Jacobian matrix $J$ of the resultant system is given by

$J=\left(\begin{array}{ccc}J_{11} & \cdots & J_{1 N} \\ \vdots & \ddots & \vdots \\ J_{N 1} & \cdots & J_{N N}\end{array}\right)$,

where $J_{i j}=\frac{\partial}{\partial a_{j}}\left(\frac{d a_{i}}{d t}-\frac{d a_{0}}{d t}\right)$, with $\frac{d a_{i}}{d t}=-\frac{1}{c_{i}} \frac{\partial u\left(a_{i}, t\right)}{\partial t}, c_{i}=$ $(-1)^{i} \frac{\partial u\left(a_{i}, t\right)}{\partial x}$ and $\frac{\partial u\left(a_{i}\right)}{\partial t}=\sum_{k=0}^{N-1} W\left(a_{i}-a_{2 k}\right)-W\left(a_{i}-a_{2 k+1}\right)$, for all $i, j \in\{1, \ldots, N\}$. If all eigenvalues have negative real part, then the solution is stable. Otherwise, if at least one eigenvalue has positive real part, then the solution is unstable.

For concreteness, consider the coupling function (6) with $A=$ $2, \alpha=\frac{\pi}{10}$ and $k=0.1$ used in the previous section. Table 1 shows the approximated solutions of system (50) for $N \in\{2,3,4,5,6\}$, when the initial values are $a_{i}=10 i$ for $i=\{1,2,3,4,5,6\}$. Table 2 displays the eigenvalues of (51) for the solutions described in Table 1. All eigenvalues have negative real part, and therefore all solutions are stable. Fig. 13 shows plots of different solutions defined by (48) where $a_{0}=0$, the values of $a_{j}$ for $j \in\{1, \ldots, N\}$ correspond to the values shown in Table 1 , and $a_{j}=a_{N}+a_{N-1}-$ $a_{2 N-1-j}$ for $j \in\{N+1, \ldots, 2 N-1\}$. The solutions represent stable $N$-bumps for $N=2, \ldots, 6$.

For the case of a two-bump solution, we can directly compare the analytical results for the width $a$ and distance $b-a$ of bumps

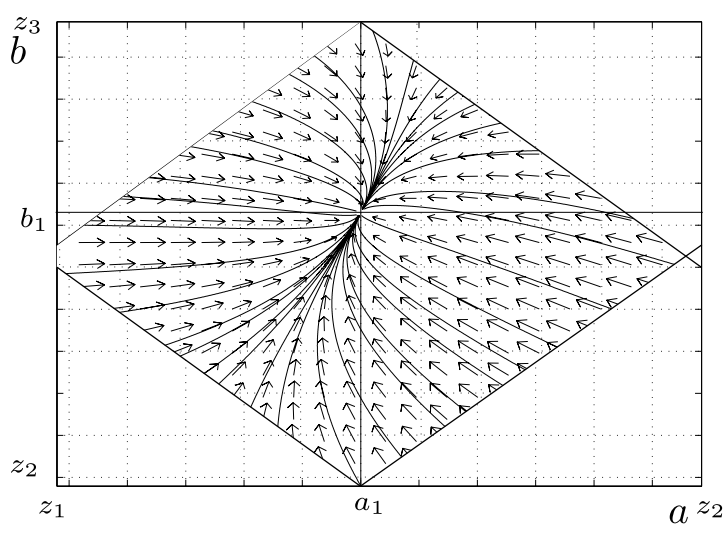

Fig. 12. Phase portrait of the planar system (33)-(34) for the example shown in Fig. 11.

Table 2

Eigenvalues of (51) for the solutions described in Table 1.

\begin{tabular}{ll}
\hline$N$ & Eigenvalues \\
\hline 2 & $\lambda_{1,2}=-0.4928 \pm 0.0888 i$ \\
3 & $\lambda_{1}=-0.5169 ; \lambda_{2,3}=-0.2031 \pm 0.1972 i$ \\
4 & $\lambda_{1,2}=-0.1964 \pm 0.0787 i \lambda_{3,4}=-0.6383 \pm 0.0347 i$ \\
5 & $\lambda_{1}=-0.0737 ; \lambda_{2}=-0.3808 ; \lambda_{3}=-0.6025 ; \lambda_{4}=-0.6922 ;$ \\
& $\lambda_{5}=-0.8029$ \\
6 & $\lambda_{1}=-0.0526 ; \lambda_{2}=-0.3057 ; \lambda_{3}=-0.5338 ; \lambda_{4}=-0.6344 ;$ \\
& $\lambda_{5}=-0.7123 ; \lambda_{6}=-0.8055$ \\
\hline
\end{tabular}

obtained in the previous section with the approximation. We know from Theorems 4 and 5 that for $h=W\left(\frac{\pi}{\alpha}\right)$ there exists a twobump solution of Eq. (1) with $a_{1}=\frac{\pi}{\alpha}$ and $b_{1}=\frac{-\arctan \left(\frac{p_{2}}{p_{3}}\right)}{\alpha}+\frac{2 \pi}{\alpha}$. For a coupling function with parameters $A=2, \alpha=\frac{\alpha}{10}$ and $k \stackrel{\alpha}{=} 0.1$, the values are $a_{1}=10$ and $b_{1}=a_{2} \approx 21.2982$. They are in perfect agreement with the values for the case $N=2$ in Table 1 . For all tested $N$, the distance between bumps is approximately constant. This confirms the observation of the numerical study in [17]. In practice, since the Newton method requires a good initial guess to converge, the values specified in Theorem 5 for the width and distance in terms of the coupling function may guide the choice of initial values for the approximation of an $\mathrm{N}$-bump solution (see Fig. 13).

\subsection{Input-induced multi-bump solutions}

In order to represent a precise multi-item memory of a series of external stimulus events, the multi-bumps in Fig. 13 should be produced by transient inputs $S_{1 b}(x)$ of a certain width $a_{s}$ and separated by a certain distance $d_{s}$. We know from Theorem 3 that the shape of $S_{1 b}(x)$ must satisfy $S_{1 b}\left(\frac{z_{1}}{2}\right)>0$ and $S_{1 b}\left(\frac{z_{2}}{2}\right)<0$. This means that the positive zero of $S_{1 b}(x)$ at position $\frac{a_{s}}{2}$ must belong to $\left(\frac{z_{1}}{2}, \frac{z_{2}}{2}\right)$, that is, $a_{s} \in\left(z_{1}, z_{2}\right)$. In the case of a bimodal input $S_{2 b}(x)$, from Theorem 6 we know that the conditions $S_{2 b}\left(\frac{z_{1}}{2}\right)<$ $0, S_{2 b}\left(\frac{z_{2}}{2}\right)>0, S_{2 b}\left(\frac{z_{3}}{2}\right)>0$ and $S_{2 b}\left(\frac{z_{4}}{2}\right)<0$ must be satisfied. This means that the two positive zeros of $S_{2 b}, \frac{d_{s}}{2}$ and $\frac{d_{s}}{2}+a_{s}$, must belong to $\left(\frac{z_{1}}{2}, \frac{z_{2}}{2}\right)$ and $\left(\frac{z_{3}}{2}, \frac{z_{4}}{2}\right)$, respectively. It follows that $d_{s} \in\left(z_{1}, z_{2}\right)$ and $d_{s}+2 a_{s} \in\left(z_{3}, z_{4}\right)$ must hold. In particular, for an equal width and distance input pattern with $a_{s}=d_{s}$ the conditions are

$a_{s} \in\left(z_{1}, z_{2}\right)$ and $3 a_{s} \in\left(z_{3}, z_{4}\right)$.

As illustrated in Fig. 14 (middle), for an input $S_{2 b}(x)$ centered at $x=\frac{3 a_{s}}{2}$, the conditions (52) are satisfied if the zeros of $S_{2 b}(x)$ are located between two successive zeros of $w(x)$. In generalization of this result, we discuss in this section computational studies of 

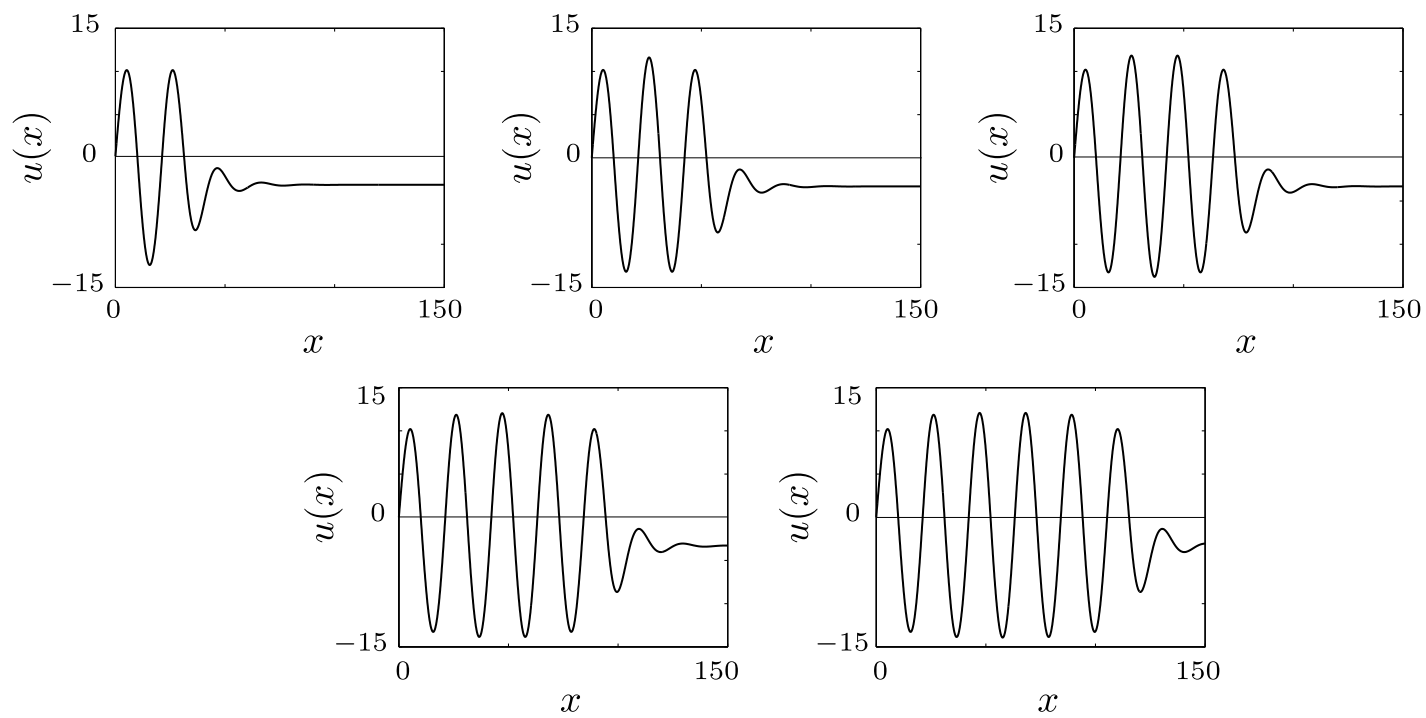

Fig. 13. $N$-bump solutions for $N=2, \ldots, 6$ obtained by solving the system (50) with $h=W(10)$. The parameters of the couplings are $A=2, k=0.1$ and $\alpha=\frac{\pi}{10}$.
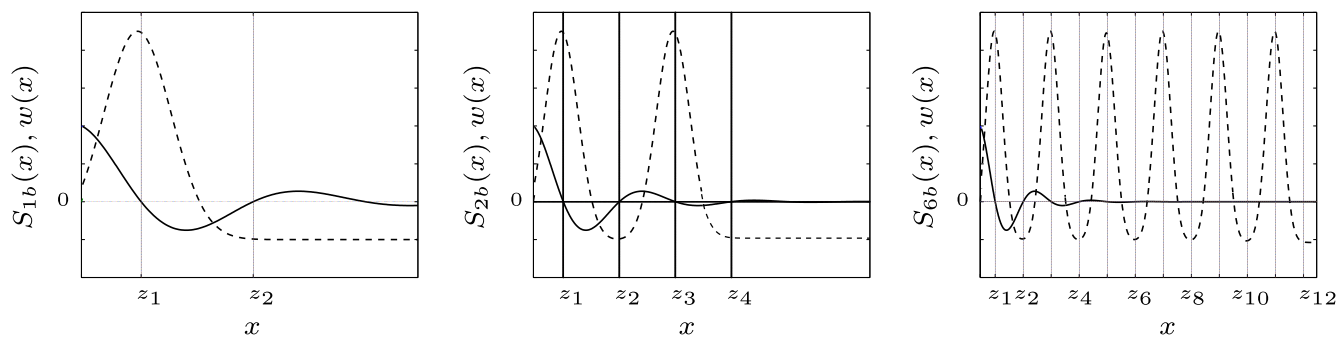

Fig. 14. Coupling function $w(x)$ (solid line) given by (6) with $A=2, k=0.1, \alpha=\frac{\pi}{10}$ and external input $S_{n b}(x)$ (dashed line) given by (53) with $S_{s}=6, S_{i}=1, \sigma=$ $5(\ln (36))^{-\frac{1}{2}}, x_{c_{j}}=20(j-1)+5$ for $j=1$ (left), $j \in 1,2$ (middle) and $j \in\{1,2,3,4,5,6\}$ (right).

the formation of $N$-bump solutions in the presence of an equal width and distance multi-modal input which is centered at $x=$ $\frac{(2 N-1) a_{s}}{2}$ and satisfies $n a_{s} \in\left(z_{n}, z_{n+1}\right), n \in 1, \ldots, N$ (Fig. 14, right). Specifically, the input pattern is given as the sum of equally spaced Gaussian functions

$S_{n b}(x)=\sum_{j=1}^{n} S_{s} e^{\left(-\frac{\left(x-x_{c_{j}}\right)^{2}}{2 \sigma^{2}}\right)}-S_{i}$,

centered at positions $x_{c_{j}}>0$.

Fig. 15 shows the formation of stationary 6-bump solutions in the presence of different input distributions (top) which converge to the same 6-bump pattern when the input is removed at time $t=$ $T$ (bottom). The 6-bump solution is triggered in the first and second examples by six inputs with the same width (approximately 11 and 6 , respectively) and the same distance between individual inputs (approximately 8 and 14, respectively), and in the third example by six inputs with constant width but varying relative distances. Note that the solution in the bottom panel corresponds to the stable 6-bump solution of system (50) shown in Fig. 13 when this pattern is centered in the interval $[30,150]$. Important for practical applications of a memory function, the numerical evidence suggests that a transient input pattern that violates the equal width and distance condition to some extent becomes represented by the stable 6 -bump solution as long as the spatial relation between the successive zeros of $w(x)$ and $S_{n b}(x)$ is preserved. A rigorous mathematical proof, like in the two-bump case (Fig. 11), of the convergence of the boundary points of each input-induced bump to the corresponding bump of the memory representation remains a challenge for the future.
Interestingly, the prediction that a neural population representation of external cues based on strong recurrent interactions within the neural population is not necessarily in perfect agreement with the perceived stimulus events is supported by neural and behavioral data $[29,30]$. A recent study reports direct experimental evidence that in multi-item working memory the individual memory traces may interfere [15]. The authors showed that depending on the their relative distance, two simultaneously memorized spatial locations are recalled with a strong bias, as if the two memory traces attract or repel each other. Such metric effects can be explained by dynamic field theory [6]. Moreover, the dynamic field model with oscillatory coupling function predicts that the mutual interactions between adjacent regions of excitation may even lead to solutions for which the number of bumps does not match the number of inputs. This is illustrated in the numerical simulations shown in Fig. 16. Here, the distance between adjacent inputs violates condition (52).

Depending on their distance, two input induced excitations may merge into a single bump at an intermediate position (column 1), may repulse each other (column 2 ), or may create an additional bump at a position without external input (column 3). While the third case illustrates the creation of a "false memory", the opposite is also true. The summed inhibition from two flanking bumps may suppress the excitation at an intermediate position if three localized inputs are close together (column 4). The mutual interactions between local excitations can be qualitatively understood by considering the excitatory or inhibitory stimulus (measured relative to the resting state) that an existing bump receives from a local excitation induced by a second input (and vice versa). If the stimulus intensity at the two boundary points 


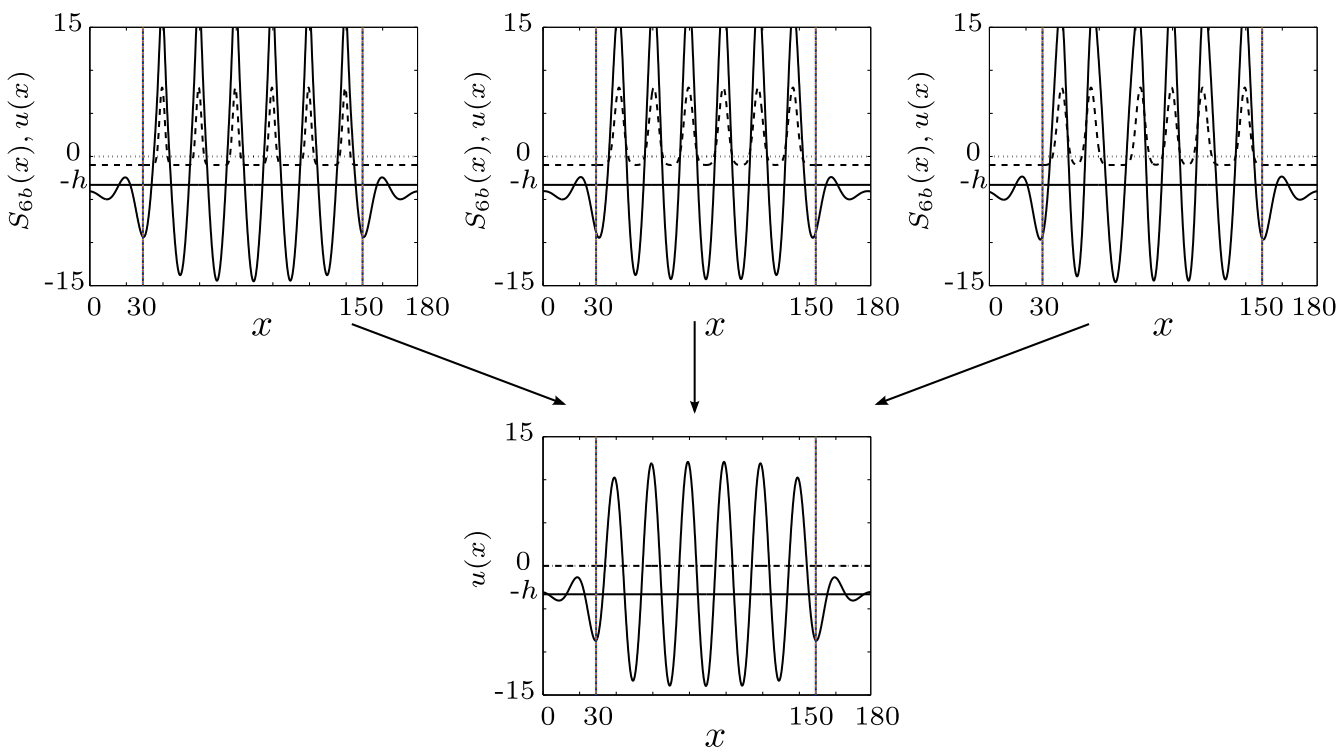

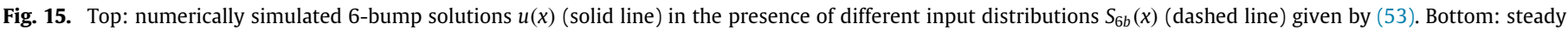

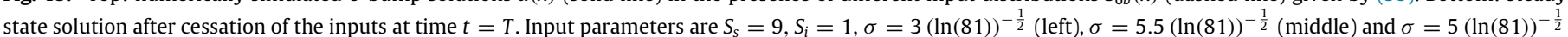

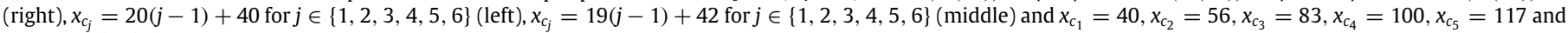
$x_{c_{6}}=140$ (right).
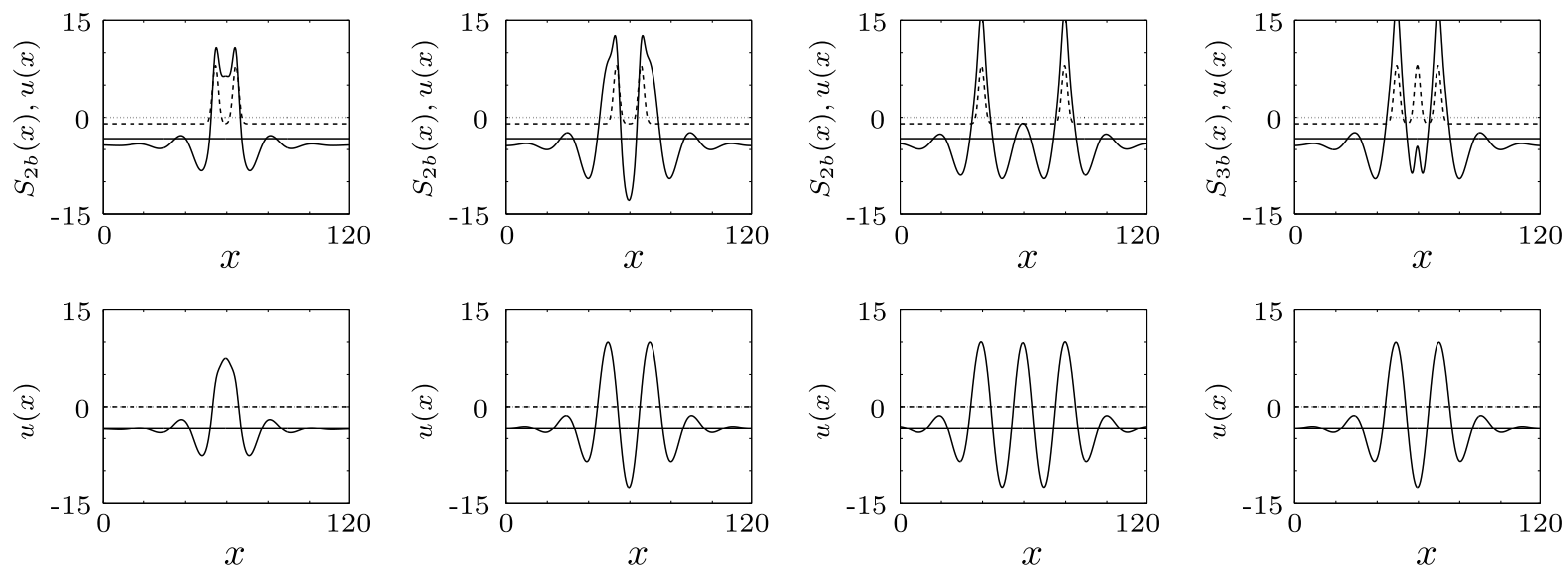

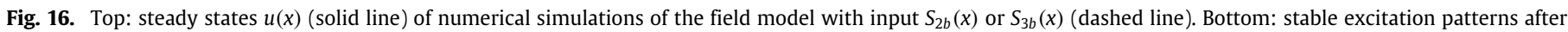

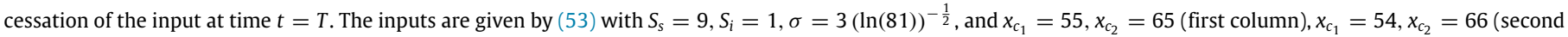
column), $x_{c_{1}}=40, x_{c_{2}}=80$ (third column), and $x_{c_{1}}=50, x_{c_{2}}=60, x_{c_{3}}=70$ (fourth column).

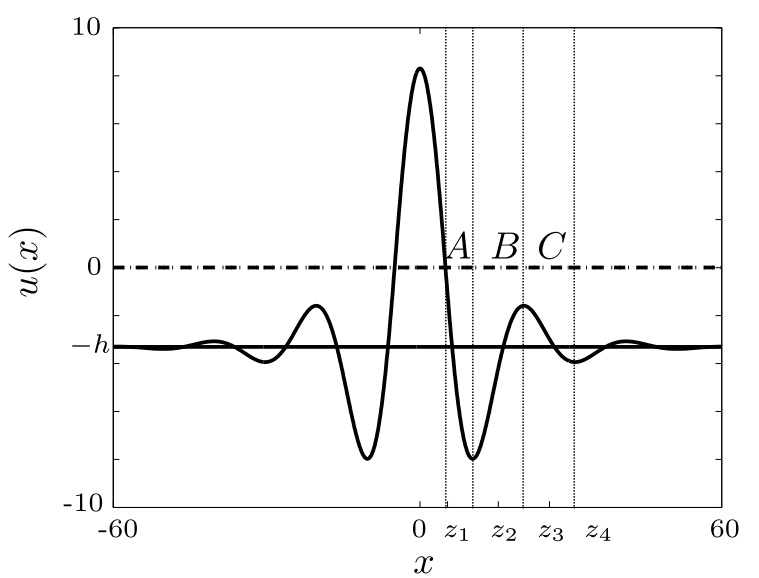

Fig. 17. Distribution of excitatory and inhibitory stimuli given by a stable bump $u(x)$. Excitation and inhibition is defined relative to the rest state $-h$. differs, the bump will start to move in the direction of the stimulus gradient [14]. When a local excitation exists in the region $A$ of another local excitation (Fig. 17), they mutually attract since the stimulus is stronger in the near sides of the local excitations than in the far sides. The equilibrium solution is a bump at an intermediate position between the input locations. For a local excitation in region $B$, the situation is opposite. The stronger stimulus at the far sides compared to the near sides causes the two excitations to move in opposite directions, until the equilibrium two-bump solution is reached. The two-bump is also the equilibrium solution when the second excitation exists in region $C$ since the two suprathreshold activity patterns again mutually attract. If two existing bumps are located at an even larger distance in which the excitatory lateral interaction in the interval $\left[z_{2}, z_{3}\right]$ overlaps, the summed excitatory stimulus at an intermediate position may be strong enough to trigger the evolution of a bump. This is the case for the example in Fig. 16 (column 3) where the distance between the flanking bumps is 40 and the maximum of the lateral excitation is located at a distance $\left(z_{2}+z_{3}\right) / 2 \approx 20$. Reducing 

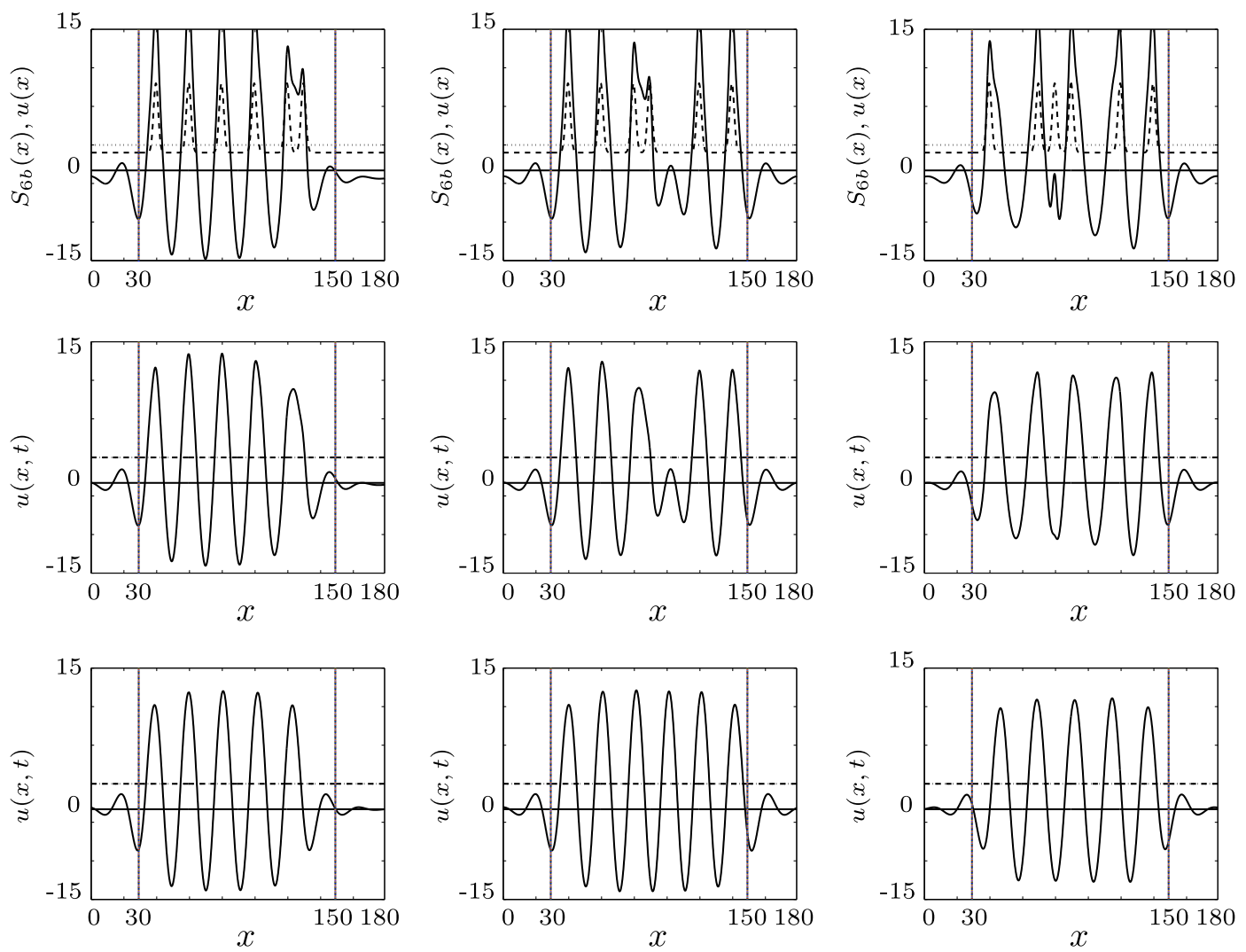

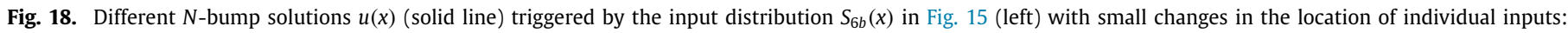

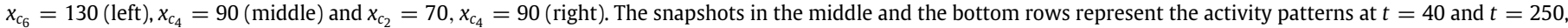
respectively, after cessation of the input. The vertical lines indicate an interval of length $L=120$.

the distance between the two flanking bumps to 20 results in the superposition of strong lateral inhibition at position $\left(z_{1}+z_{2}\right) / 2 \approx$ 10. This explains the suppression of the third input in the numerical simulation of Fig. 16 (column 4). It is important to notice that the stable solutions shown in columns 2, 3 and 4 of Fig. 16 are specific predictions of the field model with an oscillatory coupling function and cannot be explained by Amari's original model of lateral inhibition type [14]. A direct comparison of experimental findings of metric memory effects with the prediction of field models with different interaction kernels is left for future studies.

The impact of these interaction phenomena on the formation of a multi-bump pattern is illustrated in Fig. 18. The input distribution of Fig. 15 (left) has been modified by reducing the relative distance between pairs of adjacent inputs. As a result, a stable 5-bump solution evolves in response to the six inputs either because of fusion (input positions 5 and 6 , left) or suppression (input position 3 , right). The example in the middle column is particularly interesting since it shows that a stable 6-bump may evolve despite the fact that one or more input pairs in the center of the input distribution (here positions 3 and 4) violate condition (52). As can be seen in the snapshots of the temporal evolution of population activity, the local excitations at positions 3 and 4 first merge. Subsequently, a bump is created at position 4 due to the summed lateral excitation from the two flanking activity patterns.

Parameter $\alpha$ controls the spatial ranges of excitation and inhibition within the field. Consequently, the shape and the spatial extension of an $N$-bump solution vary with $\alpha$. Fig. 19 (left) compares for $N=1, \ldots, 6$ the summed width of all bumps as a function of $\alpha$. The values obtained by solving system (50) are in good agreement with the predicted value when taking $N$ times the width of a single bump (compare Fig. 3). This shows that the lateral interactions between multiple regions of excitation have a minor effect on their shapes. However, increasing the number of inputs in a given finite interval of the field is predicted to increase the probability of having distance-dependent interaction effects such as attraction, repulsion or extinction (Fig. 16, see also the discussion on memory load in [15]). Important from an application point of view is that the number of bumps that may exist in an interval of length $L$ increases with increasing $\alpha$. Fig. 19 (right) shows this dependency for the interval length $L=120$. Since the number of bumps defines the maximal number of localized inputs that can be stored in the interval, larger values of $\alpha$ increase the spatial resolution of the memory representation. Solving system (50) with $\alpha=\frac{\pi}{10}$ and $\alpha=\frac{\pi}{20}$, respectively, predicts the existence of a stable 6-bump solution and a stable 3-bump solution in an interval of length $L=120$, respectively. A comparison of the numerical simulations in Fig. 20 with the simulations in Fig. 15 shows that for $\alpha=\frac{\pi}{20}$ the six inputs trigger the evolution of a stable 2-bump solution (left) or a stable 3-bump solution (right) instead of a stable 6-bump solution obtained for $\alpha=\frac{\pi}{10}$ in Fig. 15. The decisive factor whether two or three bumps evolve is the input width. Only for the larger inputs, the initial local excitations at input locations 3 and 4 mutually attract and merge into a bump at an intermediate position. For the smaller inputs, the spread of excitation is not sufficient, and the inhibition from the flanking bumps dominates the solution.

\section{Conclusion}

In this paper, we have studied the formation of multi-bump solutions of a scalar neural field in the presence of external inputs. Persistent neural population activity, initiated by transient external cues, has been proposed as a computational mechanism in higher brain areas in order to preserve memories over time [31]. The specific research questions we have addressed are 

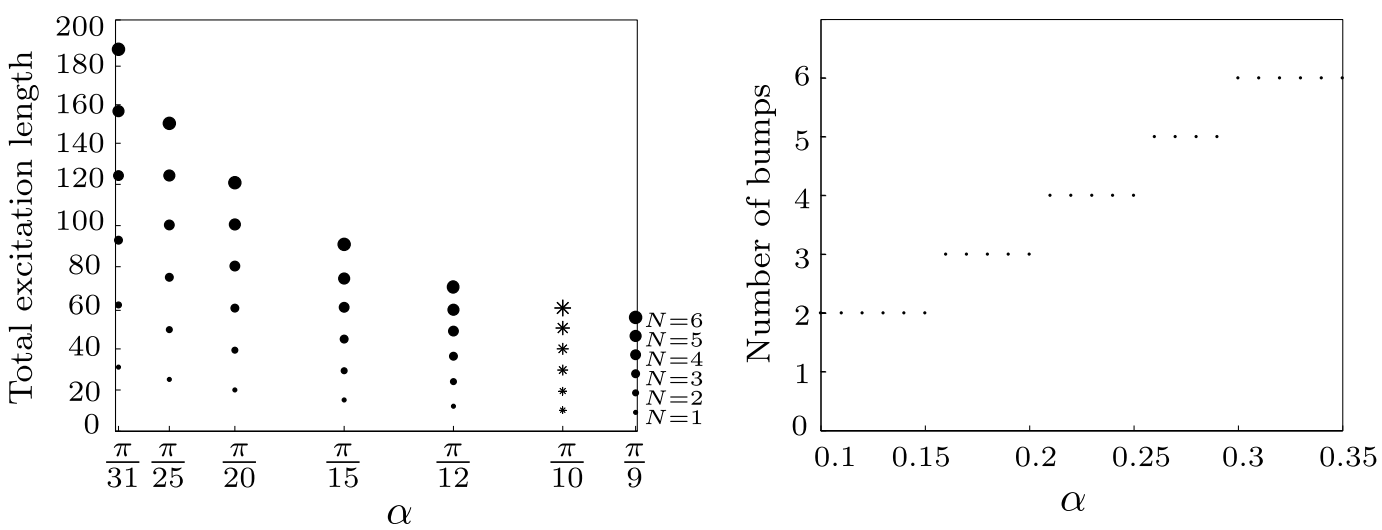

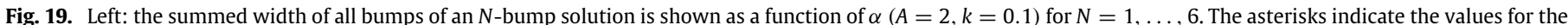
solutions shown in Fig. 13. Right: the maximum number of bumps in an interval of length $L=120$ is plotted as a function of $\alpha$.
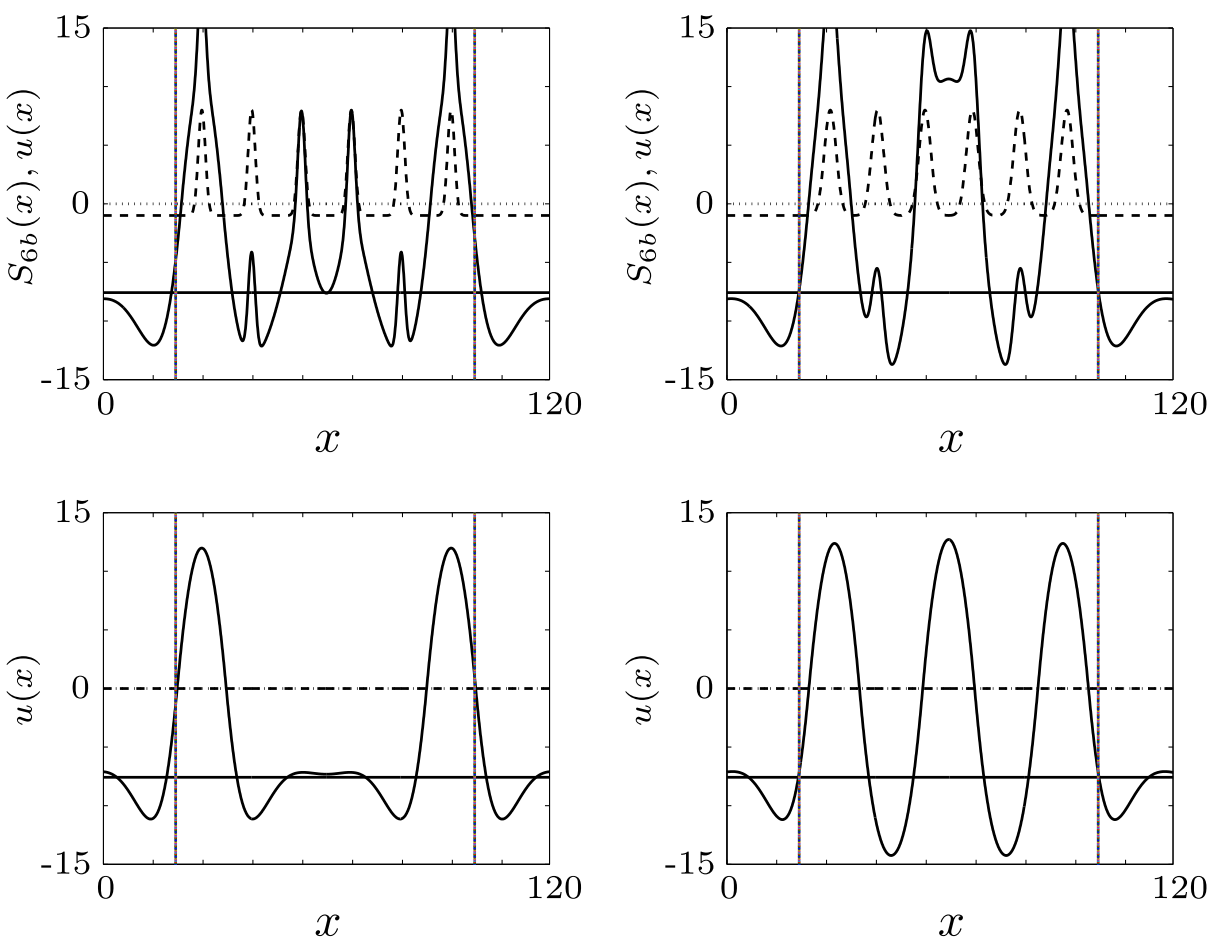

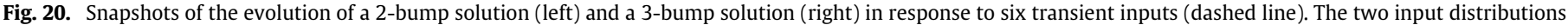
$S_{6 b}(x)$ are taken from the examples in the left and in the middle panels of Fig. 15, respectively. The value of $\alpha$ is changed to $\alpha=\frac{\pi}{20}$.

motivated by the practical need in application domains such as Neuro-Robotics [32,16] and Cognitive Sciences [10,30] to better understand the constraints on the input shape that guarantee the existence and stability of input-induced multi-bump solutions. Numerical simulations of the field model show that $N$ localized inputs will not necessarily generate a stable $N$-bump pattern even if this solution exists as an attractor state of the field dynamics.

For a class of oscillatory coupling functions $w(x)$, we developed criteria in the second and the third sections so as to ensure the existence and linear stability of one-bump and symmetric twobump solutions in a field of Amari-type without and with localized inputs. The main results of the mathematical proofs include (a) the assignment of suitable values for the parameter $h$ regarding the coupling parameters, and (b) conditions on the input width and the distance between inputs as a function of the zero crossings of $w(x)$. As an additional constraint of a memory functionality, the analysis takes into account that the input-induced unimodal or bimodal patterns should converge to a stable one-bump or two-bump solution, respectively, when the input is removed. Using phaseplane analysis tools, we have shown that for a whole range of input-depended initial values, the trajectories for the bump width $a$ and the distance between bumps $b$ converge to the equilibrium solution, thus defining the stable pattern.

In Section 5, we have extended the analysis to symmetric multi-bump solutions in a field without input by (a) deriving a set of equations that an $\mathrm{N}$-bump solution must satisfy, and (b) generalizing Amari's formal argument for bump stability. Moreover, and in numerical simulations of the field model, we have then shown that the rigorous constraints on the input shape for the two-bump case can be exploited to generate stable, input-induced multi-bump solutions.

Furthermore, for the cases where (a) the constraints on the input shape are violated, or (b) the spatial ranges of the lateral connections (controlled by the parameter $\alpha$ ) are changed, we have shown that the pattern formation process can be understood in terms of the mutual interactions between neighboring regions of excitation.

From an application perspective there are several directions that would be worthwhile exploring in future research. Multiple 
sensory inputs have to be memorized not only when they are presented simultaneously, as in the present study, but also when they are presented sequentially [10]. The evolution of a localized excitation representing a new cue thus starts from an already spatially structured initial condition (compare Fig. 17), which may effect the new population representation in many ways. The pre-structured field predicts for instance a distance-dependent detection threshold for a weaker, subthreshold cue. A thorough analysis in which the amplitude of the Gaussian input or the amplitude of the interaction kernel is treated as a bifurcation parameter would be worth performing.

A natural extension to two space dimensions with a radiallysymmetric coupling function of type (6) is of interest to implement a joint memory representation of two cue parameters. While numerical evidence reported in [17] supports the existence of some type of multi-bump solutions, a rigorous analysis of their existence, as well as their stability and spatial properties, remains an open challenge even for a two-bump solution. Nevertheless, a systematic numerical investigation of input-induced multi-bump solutions and their dependence on the spatial input properties would be of great practical value for applications of dynamic field theory.

A limitation of the Amari model is that it does not allow one to store the saliency of an external input in the bump amplitude (or their relative frequency if inputs are presented several times [6]). It would be of interest to extend the present study of multiple input-induced regions of excitation to field models with separate excitatory and inhibitory populations. These models are known to support a continuum of bump amplitudes if the strength of the recurrent interactions is precisely balanced [33].

\section{Acknowledgments}

The work received financial support from FCT through a Ph.D. grant (SFRH/BD/41179/2007) and from the EU-FP7 ITN project NETT: Neural Engineering Transformative Technologies (no. 289146).

\section{Appendix A. Proofs}

\section{A.1. Proof of Theorem 1}

Proof. If $u(x)=W\left(x+\frac{a}{2}\right)-W\left(x-\frac{a}{2}\right)-h+S_{1 b}(x)$ is a onebump solution with $R[u]=\left(-\frac{a}{2}, \frac{a}{2}\right)$, we have $u\left(-\frac{a}{2}\right)=u\left(\frac{a}{2}\right)=$ $0, u(x)>0$ on $\left(-\frac{a}{2}, \frac{a}{2}\right)$ and $u(x)<0$ otherwise. Thus, $u\left(\frac{a}{2}\right)=$ $0, u(x)>0$ if $x \in\left[0, \frac{a}{2}\right)$ and $u(x)<0$ if $x>\frac{a}{2}$. These relations can be transformed into (i)-(iii).

On the contrary, when (i)-(iii) hold, hypotheses $\left(\mathrm{H}_{1}\right)$ and $\left(\mathrm{H}_{2}\right)$ imply that $W$ is continuous and odd. Using the oddness of $W$ and hypothesis $\left(\mathrm{SH}_{1}\right)$ we have that $u(x)$ is symmetric with respect to $x=0$, that is, $u(-x)=u(x)$. Then, $u\left(-\frac{a}{2}\right)=u\left(\frac{a}{2}\right)=0, u(x)>0$ if $x \in\left(-\frac{a}{2}, 0\right]$ and $u(x)<0$ if $x<-\frac{a}{2}$. Therefore, $u\left(-\frac{a}{2}\right)=$ $u\left(\frac{a}{2}\right)=0, u(x)>0$ on $\left(-\frac{a}{2}, \frac{a}{2}\right)$ and $u(x)<0$ otherwise, that is, $u(x)$ is a one-bump solution with $R[u]=\left(-\frac{a}{2}, \frac{a}{2}\right)$.

\section{A.2. Proof of Theorem 2}

Proof. Let $W(x)$ be the integral of $w(x)$ satisfying $\left(\mathrm{H}_{1}\right),\left(\mathrm{H}_{2}\right)$ and $\left(\mathrm{H}_{5}\right)-\left(\mathrm{H}_{7}\right)$. The value of $W\left(z_{2}\right)$ is the first relative minimum of $W$ for $x>0$. From $\left(\mathrm{H}_{7}\right)$ and the fact that $W$ is odd, continuous and its oscillations decay with distance, we can conclude that $W(x)>0$ for all $x>0, W(x)<0$ for all $x<0$ and $W(0)=0$.
Assume that $W\left(z_{3}\right)<h<W\left(z_{1}\right)$ is satisfied. Let $a \in\left(z_{1}, z_{2}\right)$ be a solution of $W(a)=h$, the value of $W\left(z_{3}\right)$ is the first relative maximum of $W(x)$ for $x>a$. Then,

$W\left(x+\frac{a}{2}\right)<W(a)=h, \quad$ for all $x>\frac{a}{2}$.

From (A.1) and the fact that $W\left(x-\frac{a}{2}\right)>0$ for all $x>\frac{a}{2}$ it follows that

$u(x)=W\left(x+\frac{a}{2}\right)-W\left(x-\frac{a}{2}\right)-h<0$,

for all $x>\frac{a}{2}$.

Let $u^{\prime}(x)=w\left(x+\frac{a}{2}\right)-w\left(x-\frac{a}{2}\right)$. As the function $w(x)$ is symmetric with respect to $x=0$, positive and decreasing on $\left[0, z_{1}\right)$ and negative on $\left(z_{1}, z_{2}\right)$, and since $\frac{a}{2} \in\left(0, z_{1}\right)$ holds, we can conclude that $u^{\prime}(x)<w\left(\frac{a}{2}\right)-w\left(\frac{a}{2}\right)=0$ for all $x \in\left(0, \frac{a}{2}\right)$. Then, $u(x)$ is decreasing on $\left(0, \frac{a}{2}\right)$, and it follows that

$u(x)>u\left(\frac{a}{2}\right)=W(a)-W(0)-h=0, \quad$ when $0<x<\frac{a}{2}($ A.3 $)$

As $u(x)$ is symmetric with respect to $x=0, u\left(-\frac{a}{2}\right)=u\left(\frac{a}{2}\right)=$ $0, u(x)>0$ if $-\frac{a}{2}<x<0$ and $u(x)<0$ if $x<-\frac{a}{2}$. Therefore, $u(x)$ is a one-bump solution with $R[u]=\left(-\frac{a}{2}, \frac{a}{2}\right)$.

Since $a \in\left(z_{1}, z_{2}\right)$, we have $w(a)<0$, and therefore the solution is stable.

\section{A.3. Proof of Lemma 1}

Proof. From (9) it follows that $W\left(x+\frac{n \pi}{\alpha}\right)$, for all $n \in \mathbb{N}$, can be written as

$$
\begin{aligned}
& W\left(x+\frac{n \pi}{\alpha}\right) \\
& =-p_{1}\left(e^{-k\left(x+\frac{n \pi}{\alpha}\right)}\left(p_{3} \sin (\alpha x+n \pi)+p_{2} \cos (\alpha x+n \pi)\right)-p_{2}\right) .
\end{aligned}
$$

If $n$ is odd we have

$$
\begin{aligned}
W & \left(x+\frac{n \pi}{\alpha}\right) \\
= & -p_{1} e^{-k x} e^{-\frac{k n \pi}{\alpha}}\left(-p_{3} \sin (\alpha x)-p_{2} \cos (\alpha x)\right)+p_{1} p_{2} \\
= & -e^{-\frac{k n \pi}{\alpha}}\left(-p_{1} e^{-k x}\left(p_{3} \sin (\alpha x)+p_{2} \cos (\alpha x)\right)+p_{1} p_{2}\right) \\
& +e^{-\frac{k n \pi}{\alpha}} p_{1} p_{2}+p_{1} p_{2} \\
= & -e^{-\frac{k n \pi}{\alpha}} W(x)+p_{1} p_{2}\left(1+e^{-\frac{k n \pi}{\alpha}}\right) .
\end{aligned}
$$

On the other hand, if $n$ is even we have

$$
\begin{aligned}
W & \left(x+\frac{n \pi}{\alpha}\right) \\
= & -p_{1} e^{-k x} e^{-\frac{k n \pi}{\alpha}}\left(p_{3} \sin (\alpha x)+p_{2} \cos (\alpha x)\right)+p_{1} p_{2} \\
= & e^{-\frac{k n \pi}{\alpha}}\left(-p_{1} e^{-k x}\left(p_{3} \sin (\alpha x)+p_{2} \cos (\alpha x)\right)+p_{1} p_{2}\right) \\
& -e^{-\frac{k n \pi}{\alpha}} p_{1} p_{2}+p_{1} p_{2} \\
= & e^{-\frac{k n \pi}{\alpha}} W(x)+p_{1} p_{2}\left(1-e^{-\frac{k n \pi}{\alpha}}\right) .
\end{aligned}
$$

This proves the first equality, the second equality can be proven in a similar manner.

\section{A.4. Proof of Lemma 2}

Proof. By Lemma 1 we obtain

$$
W\left(\frac{\pi}{\alpha}\right)=W\left(0+\frac{\pi}{\alpha}\right)=-e^{-\frac{k \pi}{\alpha}} W(0)+p_{1} p_{2}\left(1+e^{-\frac{k \pi}{\alpha}}\right) ;
$$


and

$W\left(z_{3}\right)=W\left(z_{2}+\frac{\pi}{\alpha}\right)=-e^{-\frac{k \pi}{\alpha}} W\left(z_{2}\right)+p_{1} p_{2}\left(1+e^{-\frac{k \pi}{\alpha}}\right)$.

As $W(0)=0$,

$W\left(\frac{\pi}{\alpha}\right)=p_{1} p_{2}\left(1+e^{-\frac{k \pi}{\alpha}}\right)$.

Suppose that $W\left(z_{2}\right)>0$, then $-e^{-\frac{k \pi}{\alpha}} W\left(z_{2}\right)<0$ and

$p_{1} p_{2}\left(1+e^{-\frac{k \pi}{\alpha}}\right)>-e^{-\frac{k \pi}{\alpha}} W\left(z_{2}\right)+p_{1} p_{2}\left(1+e^{-\frac{k \pi}{\alpha}}\right)$

i.e.,

$W\left(\frac{\pi}{\alpha}\right)>W\left(z_{3}\right)$.

\section{A.5. Proof of Theorem 3}

Proof. From $\left(\mathrm{H}_{2}\right)$ and $\left(\mathrm{SH}_{1}\right)$, the function defined by $G(x)=$ $W(x)+S_{1 b}\left(\frac{x}{2}\right)-h$ is continuous for all $x \geq 0$.

Assuming that $S_{1 b}\left(\frac{z_{1}}{2}\right)>0$ and $h<W\left(z_{1}\right)$ hold, we obtain

$W\left(z_{1}\right)+S_{1 b}\left(\frac{z_{1}}{2}\right)-h>0$.

On the other hand, assuming that $S_{1 b}\left(\frac{z_{2}}{2}\right)<0$ and $h>W\left(z_{3}\right)$ hold, we obtain

$W\left(z_{2}\right)+S_{1 b}\left(\frac{z_{2}}{2}\right)-h<0$.

Thus, by the intermediate value theorem, there exists a value $a \in$ $\left(z_{1}, z_{2}\right)$ such that $G(a)=0$. As $G(x)$ is monotonically decreasing in the interval $\left[z_{1}, z_{2}\right]$ there exists a unique value $a \in\left(z_{1}, z_{2}\right)$, that satisfies the equation $G(x)=0$. In addition, if $x \in\left[0, z_{1}\right]$, we have $h-S_{1 b}\left(\frac{x}{2}\right)-W(x)<0$ because $h-S_{1 b}\left(\frac{x}{2}\right)-W(x)$ is decreasing and $S_{1 b}(0)>h$. If $x \geq z_{2}$, we have $h-S_{1 b}\left(\frac{x}{2}\right)-W(x)>0$ because $h>W(x), S_{1 b}\left(\frac{x}{2}\right)$ is decreasing and $S_{1 b}\left(\frac{z_{2}}{2}\right)<0$. Therefore the equation

$h-S_{1 b}\left(\frac{x}{2}\right)=W(x)$

has a unique positive solution $a$ that belongs to $\left(z_{1}, z_{2}\right)$.

\section{A.6. Proof of Theorem 4}

If $h=W\left(\frac{\pi}{\alpha}\right)$ (with $W\left(\frac{\pi}{\alpha}\right)=p_{1} p_{2}\left(1+e^{-\frac{k \pi}{\alpha}}\right)>0$ ), and $\tau$ is a triple of the form $(a, b, a+b)$, then $u_{\tau}$ is a $a$-quasi-solution if and only if $u_{\tau}(0)=u_{\tau}(a+b)=0$ and $u_{\tau}(a)=u_{\tau}(b)=0$. Next, we find a non-trivial solution of the following system of equations

$W(a+b)-W(b)=0$,

and

$W(b)-W(b-a)=0$.

Let $a=\frac{\pi}{\alpha}$, it follows that

$W\left(\frac{\pi}{\alpha}+b\right)-W(b)=0$,

and

$W(b)-W\left(b-\frac{\pi}{\alpha}\right)=0$.

Using (9), and by Lemma 1, we obtain

$-e^{-\frac{k \pi}{\alpha}} W(b)+p_{1} p_{2}\left(1+e^{-\frac{k \pi}{\alpha}}\right)-W(b)=0$, and

$W(b)+e^{\frac{k \pi}{\alpha}} W(b)-p_{1} p_{2}\left(1+e^{\frac{k \pi}{\alpha}}\right)=0$.

It follows

$W(b)=p_{1} p_{2}$.

Since $b>a$, and $W(b)=p_{1} p_{2}, p_{3}<0$, we obtain

$b=-\frac{\arctan \left(\frac{p_{2}}{p_{3}}\right)}{\alpha}+\frac{n \pi}{\alpha}$ for some $n \in \mathbb{N}$.

Thus, for $a=\frac{\pi}{\alpha}$ there exists a value of $b$ such that the conditions (A.7) and (A.8) are satisfied. This completes the proof of the theorem.

\section{A.7. Proof of Theorem 5}

Proof. Recall that

$$
\begin{aligned}
u(x)= & W\left(x+\frac{b+a}{2}\right)-W\left(x+\frac{b-a}{2}\right) \\
& +W\left(x-\frac{b-a}{2}\right)-W\left(x-\frac{b+a}{2}\right)-W\left(\frac{\pi}{\alpha}\right)
\end{aligned}
$$

and

$u_{\tau}(x)=W(x)-W(x-a)+W(x-b)-W(x-c)-h$.

If $a=\frac{\pi}{\alpha}, b \in\left(z_{2}, z_{3}\right)$ such that $W(b)=p_{1} p_{2}, c=a+b$, with $h=W\left(\frac{\pi}{\alpha}\right)$, by Theorem 4, we have that $u_{\tau}(0)=u_{\tau}\left(\frac{\pi}{\alpha}\right)=$ $u_{\tau}(b)=u_{\tau}\left(b+\frac{\pi}{\alpha}\right)=0$, which is equivalent to $u\left(-\frac{b}{2}-\frac{\pi}{2 \alpha}\right)=$ $u\left(-\frac{b}{2}+\frac{\pi}{2 \alpha}\right)=u\left(\frac{b}{2}-\frac{\pi}{2 \alpha}\right)=u\left(\frac{b}{2}+\frac{\pi}{2 \alpha}\right)=0$.

As $b \in\left(z_{2}, z_{3}\right)$, and since $W(b)=W\left(b+\frac{(n-3) \pi}{\alpha}\right)=p_{1} p_{2}$ for all $n \in \mathbb{N}$, we have for all $x \in\left[b+\frac{(n-3) \pi}{\alpha}, b+\frac{(n-2) \pi}{\alpha}\right]$

$W(x) \geq p_{1} p_{2}, \quad$ if $n$ is odd,

and

$W(x) \leq p_{1} p_{2}, \quad$ if $n$ is even.

(i) If $0<x<\frac{b}{2}-\frac{\pi}{2 \alpha}$, we have

$x-\frac{b}{2}+\frac{\pi}{2 \alpha}<0$

$x-\frac{b}{2}-\frac{\pi}{2 \alpha}<-\frac{\pi}{\alpha}$

$b-\frac{2 \pi}{\alpha}<\frac{b}{2}-\frac{\pi}{2 \alpha}<x+\frac{b}{2}-\frac{\pi}{2 \alpha}<b-\frac{\pi}{\alpha}$,

and

$b-\frac{\pi}{\alpha}<\frac{b}{2}+\frac{\pi}{2 \alpha}<x+\frac{b}{2}+\frac{\pi}{2 \alpha}<b$.

Then, from (A.19) and (A.20), it follows

$W\left(x-\frac{b}{2}+\frac{\pi}{2 \alpha}\right)<0$ and

$W\left(x-\frac{b}{2}-\frac{\pi}{2 \alpha}\right)>-W\left(\frac{\pi}{\alpha}\right)$.

On the other hand, from (A.17), (A.18), (A.21) and (A.22) it follows

$W\left(x+\frac{b}{2}-\frac{\pi}{2 \alpha}\right)>p_{1} p_{2}$ and

$W\left(x+\frac{b}{2}+\frac{\pi}{2 \alpha}\right)<p_{1} p_{2}$. 
Then, from (A.23) and (A.24), we conclude that

$u(x)<p_{1} p_{2}-p_{1} p_{2}-0+W\left(\frac{\pi}{\alpha}\right)-W\left(\frac{\pi}{\alpha}\right)=0$.

Therefore, $u(x)<0$ when $0<x<\frac{b}{2}-\frac{\pi}{2 \alpha}$.

(ii) Let $F(x)=W\left(x-\frac{b}{2}+\frac{\pi}{2 \alpha}\right)-W\left(x-\frac{b}{2}-\frac{\pi}{2 \alpha}\right)$. It follows from our hypotheses that $F(x)$ is a $C^{1}$ function on $[0,+\infty)$ with

$F^{\prime}(x)=w\left(x-\frac{b}{2}+\frac{\pi}{2 \alpha}\right)-w\left(x-\frac{b}{2}-\frac{\pi}{2 \alpha}\right)$.

As the function $w(x)$ is symmetric with respect to $x=0$, positive and decreasing on $\left[0, z_{1}\right)$ and negative on $\left(z_{1}, z_{2}\right)$, and since $\frac{\pi}{2 \alpha} \in\left(0, z_{1}\right)$ holds, we can conclude that $F^{\prime}(x) \geq$ $w\left(\frac{\pi}{2 \alpha}\right)-w\left(\frac{\pi}{2 \alpha}\right)=0$ for all $x \in\left[\frac{b}{2}-\frac{\pi}{2 \alpha}, \frac{b}{2}\right]$ and $F^{\prime}(x) \leq$ $w\left(\frac{\pi}{2 \alpha}\right)-w\left(\frac{\pi}{2 \alpha}\right)=0$ for all $x \in\left[\frac{b}{2}, \frac{b}{2}+\frac{\pi}{2 \alpha},\right]$. Then, $F(x)$ is increasing on $\left[\frac{b}{2}-\frac{\pi}{2 \alpha}, \frac{b}{2}\right]$ and decreasing on $\left[\frac{b}{2}, \frac{b}{2}+\frac{\pi}{2 \alpha}\right]$. It follows that

$F(x) \geq W(0)-W\left(-\frac{\pi}{\alpha}\right) \quad$ if $x \in\left[\frac{b}{2}-\frac{\pi}{2 \alpha}, \frac{b}{2}\right]$,

and

$F(x) \geq W\left(\frac{\pi}{\alpha}\right)-W(0) \quad$ if $x \in\left[\frac{b}{2}, \frac{b}{2}+\frac{\pi}{2 \alpha}\right]$.

Thus,

$F(x) \geq W\left(\frac{\pi}{\alpha}\right), \quad$ if $\frac{b}{2}-\frac{\pi}{2 \alpha}<x<\frac{b}{2}+\frac{\pi}{2 \alpha}$.

If $x \in\left[\frac{b}{2}-\frac{\pi}{2 \alpha}, \frac{b}{2}+\frac{\pi}{2 \alpha}\right]$, from (A.17) and (A.18) it follows

$W\left(x+\frac{b}{2}+\frac{\pi}{2 \alpha}\right)-W\left(x+\frac{b}{2}-\frac{\pi}{2 \alpha}\right)$

$>p_{1} p_{2}-p_{1} p_{2}=0$.

Then, from (A.29) and (A.30) we obtain

$u(x)>W\left(\frac{\pi}{\alpha}\right)+0-W\left(\frac{\pi}{\alpha}\right)=0$.

Therefore, $u(x)>0$ if $\frac{b}{2}-\frac{\pi}{2 \alpha}<x<\frac{b}{2}+\frac{\pi}{2 \alpha}$.

(iii) If $\frac{b}{2}+\frac{\pi}{2 \alpha}<x \leq \frac{b}{2}+\frac{3 \pi}{2 \alpha}$, we have $W\left(x-\frac{b}{2}-\frac{\pi}{2 \alpha}\right)>0$, from (A.17) and (A.18) it follows that $W\left(x+\frac{b}{2}+\frac{\pi}{2 \alpha}\right) \leq p_{1} p_{2}$ and that $W\left(x+\frac{b}{2}-\frac{\pi}{2 \alpha}\right) \geq p_{1} p_{2}$. From Lemma 2 we have $W\left(x-\frac{b}{2}+\frac{\pi}{2 \alpha}\right)<W\left(\frac{\pi}{\alpha}\right)$. Thus, we obtain

$u(x)<p_{1} p_{2}-p_{1} p_{2}+W\left(\frac{\pi}{\alpha}\right)-0-W\left(\frac{\pi}{\alpha}\right)=0$.

If $x>\frac{b}{2}+\frac{3 \pi}{2 \alpha}$, we have $W\left(x+\frac{b}{2}+\frac{\pi}{2 \alpha}\right)<W\left(z_{5}\right), W\left(x+\frac{b}{2}-\right.$ $\left.\frac{\pi}{2 \alpha}\right)>W\left(z_{4}\right), W\left(x-\frac{b}{2}+\frac{\pi}{2 \alpha}\right)<W\left(z_{3}\right)$, and $W\left(x-\frac{b}{2}-\frac{\pi}{2 \alpha}\right)$ $>W\left(z_{2}\right)$, consequently we obtain

$u(x)<W\left(z_{5}\right)-W\left(z_{4}\right)+W\left(z_{3}\right)-W\left(z_{2}\right)-W\left(\frac{\pi}{\alpha}\right)$.

By Lemma 1, it follows that

$$
\begin{aligned}
u(x)< & -\left(e^{-\frac{3 k \pi}{\alpha}}+e^{-\frac{2 k \pi}{\alpha}}\right)\left(1+e^{\frac{2 k \pi}{\alpha}}\right) W\left(z_{2}\right) \\
& +p_{1} p_{2}\left(e^{-\frac{3 k \pi}{\alpha}}+e^{-\frac{2 k \pi}{\alpha}}\right),
\end{aligned}
$$

from $\left(\mathrm{H}_{8}\right)$, we obtain

$$
\begin{aligned}
u(x)< & -\left(e^{-\frac{3 k \pi}{\alpha}}+e^{-\frac{2 k \pi}{\alpha}}\right) p_{1} p_{2} \\
& +p_{1} p_{2}\left(e^{-\frac{3 k \pi}{\alpha}}+e^{-\frac{2 k \pi}{\alpha}}\right)=0 .
\end{aligned}
$$

Therefore, from (A.32) and (A.35), $u(x)<0$ for all $x>\frac{b}{2}+\frac{\pi}{2 \alpha}$.
As $u(x)$ is symmetric with respect to $x=0$, from (i)-(iii) we can conclude that $u(x)<0$ if $-\frac{b}{2}+\frac{\pi}{2 \alpha}<x<0, u(x)>0$ if $-\frac{b}{2}-\frac{\pi}{2 \alpha}<x<-\frac{b}{2}+\frac{\pi}{2 \alpha}$, and $u(x)<0$ if $x<-\frac{b}{2}-\frac{\pi}{2 \alpha}$.

Therefore, we can conclude that $u(x)$ represents a two-bump solution with $R[u]=\left(-\frac{b}{2}-\frac{\pi}{2 \alpha},-\frac{b}{2}+\frac{\pi}{2 \alpha}\right) \cup\left(\frac{b}{2}-\frac{\pi}{2 \alpha}, \frac{b}{2}+\frac{\pi}{2 \alpha}\right)$.

We recall from (19) that the solution corresponding to $(a, b)=$ $\left(\frac{\pi}{\alpha}, b\right)$ is stable if $b \in\left(z_{2 n}, z_{2 n+1}\right)$ for some $n \in \mathbb{N}$. Since $b \in\left(z_{2}, z_{3}\right)$, we conclude that the solution is stable with respect to perturbations that preserve the equal-width condition. This completes the proof of the theorem.

\section{A.8. Proof of Theorem 6}

Proof. We define

$$
\begin{aligned}
F_{1}(x, y)= & W(x)+W(y)-W(y-x)-W\left(\frac{\pi}{\alpha}\right) \\
& +S_{2 b}\left(\frac{y-x}{2}\right)
\end{aligned}
$$

and

$$
\begin{aligned}
F_{2}(x, y)= & W(x)-W(y)+W(x+y)-W\left(\frac{\pi}{\alpha}\right) \\
& +S_{2 b}\left(\frac{x+y}{2}\right) .
\end{aligned}
$$

First consider the line $y=x+z_{1}$ for $\frac{\pi}{\alpha} \leq x<z_{2}$ and the line $y=x+z_{2}$ for $z_{1}<x \leq \frac{\pi}{\alpha}$ (compare Fig. 10). Substituting $y$ in (A.36) by $x+z_{1}$ and $x+z_{2}$, respectively, we obtain

$F_{1}\left(x, x+z_{1}\right)=W(x)+W\left(x+z_{1}\right)-W\left(z_{1}\right)$

$$
-W\left(\frac{\pi}{\alpha}\right)+S_{2 b}\left(\frac{z_{1}}{2}\right)
$$

and

$$
\begin{aligned}
F_{1}\left(x, x+z_{2}\right)= & W(x)+W\left(x+z_{2}\right)-W\left(z_{2}\right) \\
& -W\left(\frac{\pi}{\alpha}\right)+S_{2 b}\left(\frac{z_{2}}{2}\right) .
\end{aligned}
$$

Since $S_{2 b}\left(\frac{z_{1}}{2}\right)<0$ by hypothesis, $W(x)<W\left(\frac{\pi}{\alpha}\right)$ and $W\left(x+z_{1}\right)<W\left(z_{1}\right)$ for all $x \in\left[\frac{\pi}{\alpha}, z_{2}\right)$, we conclude that

$F_{1}\left(x, x+z_{1}\right)<0$ for all $x \in\left[\frac{\pi}{\alpha}, z_{2}\right)$.

On the other hand, since $S_{2 b}\left(\frac{z_{2}}{2}\right)>0$ by hypothesis, $W(x)>$ $W\left(\frac{\pi}{\alpha}\right)$ and $W\left(x+z_{2}\right)>W\left(z_{2}\right)$ for all $x \in\left(z_{1}, \frac{\pi}{\alpha}\right]$, we conclude that

$F_{1}\left(x, x+z_{2}\right)>0$ for all $x \in\left(z_{1}, \frac{\pi}{\alpha}\right]$.

Thus, on the line connecting the two points $P_{1}=\left(x_{1}, x_{1}+z_{1}\right)$ and $P_{2}=\left(x_{2}, x_{2}+z_{2}\right)$ with $x_{1} \in\left[\frac{\pi}{\alpha}, z_{2}\right)$ and $x_{2} \in\left(z_{1}, \frac{\pi}{\alpha}\right]$, there exists a point $\left(x^{*}, y^{*}\right)$ such that $F_{1}\left(x^{*}, y^{*}\right)=0$. In addition, $\frac{\partial F_{1}}{\partial y}(x, y)>0$ holds in the region

$\Omega_{1}=\left\{(x, y) \in \mathbb{R}^{2} \mid x+z_{1}<y<x+z_{2} \wedge r_{1}<y<r_{2}\right\}$,

where $r_{1}, r_{2}$ are the lines defined by the points $P_{1}$ and $P_{2}$ with $x_{1}=\frac{\pi}{\alpha}, x_{2}=z_{1}$, and $x_{1}=z_{2}, x_{2}=\frac{\pi}{\alpha}$, respectively. Therefore, by the implicit function theorem, the equation $F_{1}(x, y)=0$ defines $y$ implicitly as an increasing function of $x$ in the region $\Omega_{1}$.

Now, consider the line $y=-x+z_{3}$ for $z_{1}<x \leq \frac{\pi}{\alpha}$ and the line $y=-x+z_{4}$ for $\frac{\pi}{\alpha} \leq x<z_{2}$. Substituting $y$ in (A.37) by $-x+z_{3}$ and $-x+z_{4}$, respectively, we obtain

$F_{2}\left(x,-x+z_{3}\right)=W(x)-W\left(-x+z_{3}\right)+W\left(z_{3}\right)$

$$
-W\left(\frac{\pi}{\alpha}\right)+S_{2 b}\left(\frac{z_{3}}{2}\right)
$$


and

$$
\begin{gathered}
F_{2}\left(x,-x+z_{4}\right)=W(x)-W\left(-x+z_{4}\right)+W\left(z_{4}\right) \\
-W\left(\frac{\pi}{\alpha}\right)+S_{2 b}\left(\frac{z_{4}}{2}\right) .
\end{gathered}
$$

Since $S_{2 b}\left(\frac{z_{3}}{2}\right)>0$ by hypothesis, $W\left(z_{3}\right)>W\left(-x+z_{3}\right)$ and $W(x)>W\left(\frac{\pi}{\alpha}\right)$ for all $x \in\left(z_{1}, \frac{\pi}{\alpha}\right]$, we conclude that

$F_{2}\left(x,-x+z_{3}\right)>0$ for all $x \in\left(z_{1}, \frac{\pi}{\alpha}\right]$.

On the other hand, $S_{2 b}\left(\frac{z_{4}}{2}\right)<0$ by hypothesis, $W(x)<W\left(\frac{\pi}{\alpha}\right)$ and $W\left(z_{4}\right)<W\left(-x+z_{4}\right)$ for all $x \in\left[\frac{\pi}{\alpha}, z_{2}\right)$, we have

$F_{2}\left(x,-x+z_{4}\right)<0$ for all $x \in\left[\frac{\pi}{\alpha}, z_{2}\right)$.

Thus, on the line connecting the two points $P_{3}=\left(x_{3},-x_{3}+z_{3}\right)$ and $P_{4}=\left(x_{4},-x_{4}+z_{4}\right)$ with $x_{3} \in\left(z_{1}, \frac{\pi}{\alpha}\right]$ and $x_{4} \in\left[\frac{\pi}{\alpha}, z_{2}\right)$, there exists a point $\left(x^{*}, y^{*}\right)$ such that $F_{2}\left(x^{*}, y^{*}\right)=0$. In addition, $\frac{\partial F_{2}}{\partial y}(x, y)<0$ holds. Thus, the equation $F_{2}(x, y)=0$ defines $y$ implicitly as an increasing function of $x$ in the region

$$
\begin{aligned}
\Omega_{2}= & \left\{(x, y) \in \mathbb{R}^{2} \mid-x+z_{3}<y<-x+z_{4}\right. \\
& \left.\wedge r_{3}<y<r_{4}\right\},
\end{aligned}
$$

where $r_{3}, r_{4}$ are the lines defined by points $P_{3}$ and $P_{4}$ with $x_{3}=$ $\frac{\pi}{\alpha}, x_{4}=z_{1}$ and $x_{3}=z_{2}, x_{4}=\frac{\pi}{\alpha}$, respectively.

Therefore, we can conclude that there exists a unique point $(a, b) \in \Omega_{1} \cap \Omega_{2} \subset \Omega$ that satisfies the system of equations $F_{1}(a, b)=0$ and $F_{2}(a, b)=0$ (compare Fig. 11).

\section{A.9. Proof of Proposition 1}

Proof. From (48) we can write

$u\left(a_{N+m}\right)=\sum_{i=0}^{N-1}\left(W\left(a_{N+m}-a_{2 i}\right)-W\left(a_{N+m}-a_{2 i+1}\right)\right)-h$,

$m \in\{1, \ldots, N-1\}$.

As $u$ is symmetric with respect to the point $\frac{a_{0}+a_{2 N-1}}{2}$, we have $a_{N+m}=a_{N}+a_{N-1}-a_{N-(1+m)}, m \in\{1, \ldots, N-1\}^{2}$. Then,

$$
\begin{aligned}
u\left(a_{N+m}\right)= & \sum_{i=0}^{N-1}\left(W\left(a_{N}+a_{N-1}-a_{N-(1+m)}-a_{2 i}\right)\right. \\
& \left.-W\left(a_{N}+a_{N-1}-a_{N-(1+m)}-a_{2 i+1}\right)\right)-h .
\end{aligned}
$$

As $a_{N+m}=a_{N}+a_{N-1}-a_{N-(1+m)}$, for $m=N-1-2 i$ and $m=N-2-2 i$, we have

$a_{N}+a_{N-1}-a_{2 i}=a_{2(N-i)-1}$

and

$a_{N}+a_{N-1}-a_{2 i+1}=a_{2(N-i-1)}$,

respectively. Thus, we obtain

$$
\begin{aligned}
u\left(a_{N+m}\right)= & \sum_{i=0}^{N-1}\left(W\left(a_{2(N-i)-1}-a_{N-(1+m)}\right)\right. \\
& \left.-W\left(a_{2(N-i-1)}-a_{N-(1+m)}\right)\right)-h .
\end{aligned}
$$

Using the oddness of $W(x)$, it follows that

$$
\begin{aligned}
u\left(a_{N+m}\right)= & \sum_{i=0}^{N-1}\left(-W\left(a_{N-(1+m)}-a_{2 i+1}\right)\right. \\
& \left.+W\left(a_{N-(1+m)}-a_{2 i}\right)\right)-h,
\end{aligned}
$$

$m \in\{1, \ldots, N-1\}$.
Therefore,

$u\left(a_{N+m}\right)=u\left(a_{N-(1+m)}\right)$

and the system (49) can be reduced to

$\left\{\begin{array}{l}u\left(a_{0}\right)=0 \\ u\left(a_{1}\right)=0 \\ \cdots \\ u\left(a_{N}\right)=0 .\end{array}\right.$

\section{Appendix B. Numerical method and parameters}

Using Matlab [25], we implement a forward Euler scheme to integrate the neural field equation

$\frac{\partial u(x, t)}{\partial t}=-u(x, t)+\int_{-\infty}^{\infty} w(x-y) f(u(y, t)) d y+g(x, t)$,

where $g(x, t)=S(x, t)-h$, to a steady state.

We assume a finite domain $\Omega$ with length $L$. To minimize the effect of the boundaries on the pattern formation process, the domain size is chosen much larger than the length of the input distribution, and the boundary points evolve freely according to the numerical scheme. $\Omega$ is discretized into $n$ equal intervals of size $\Delta_{x}$ so that $\Delta_{x}=\frac{L}{n}$. The spatial discretization defines the position of neurons labeled as $x_{i}=i \Delta_{x}$ for $i=0,1, \ldots, n$. To find $u(x, T)$ where $T>0$, we discretize time $T$ into $m$ equal steps of size $\Delta_{t}$ and write $t_{j}=j \Delta_{t}$ for $j=0,1, \ldots, m$. The derivative in (B.1) is replaced by the forward difference approximation

$u_{t}=\frac{v_{i, j+1}-v_{i, j}}{\Delta_{t}}+O\left(\Delta_{t}\right)$,

where $v_{i, j}$ denotes the approximation of $u\left(x_{i}, t_{j}\right)=u\left(i \Delta_{x}, j \Delta_{t}\right)$. Ignoring the integral term, we have the scheme

$v_{i, j+i}=\left(1-\Delta_{t}\right) v_{i, j} \Delta_{t} g\left(x_{i}, t_{j}\right)$.

To solve Eq. (B.1) numerically, the nonlinear term corresponding to the convolution integral is evaluated at step $j$ using the Matlab function conv with open boundary conditions. Note that the convolution can be more effectively performed with a fast Fourier transform. The forward Euler scheme is then given by

$v_{i, j+i}=\left(1-\Delta_{t}\right) v_{i, j}+\Delta_{t}\left(g\left(x_{i}, t_{j}\right)+W_{j}\right)$,

where $W_{j}$ denotes the evaluation of the nonlinear integral term at time step $j$.

For the numerical simulations presented in this paper we applied $\Delta_{x}=0.005$ and $\Delta_{t}=0.05$.

\section{References}

[1] S. Coombes, Waves, bumps, and patterns in neural field theories, Biol. Cybernet. 93 (2) (2005) 91-108.

[2] G.B. Ermentrout, S.E. Folias, Z.P. Kilpatrick, Spatiotemporal pattern formation in neural fields with linear adaptation, in: Neural Fields, Springer, 2014, pp. $119-151$.

[3] K. Zhang Representation of spatial orientation by the intrinsic dynamics of the head-direction cell ensemble: a theory, J. Neurosci. 16 (6) (1996) 2112-2126.

[4] M. Camperi, X.-J. Wang, A model of visuospatial working memory in prefrontal cortex: recurrent network and cellular bistability, J. Comput. Neurosci. 5 (4) (1998) 383-405.

[5] E. Bicho, P. Mallet, G. Schöner, Target representation on an autonomous vehicle with low-level sensors, Int. J. Robot. Res. 19 (5) (2000) 424-447.

[6] W. Erlhagen, G. Schöner, Dynamic field theory of movement preparation, Psychol. Rev. 109 (3) (2002) 545-572.

[7] A.R. Schutte, J.P. Spencer, G. Schöner, Testing the dynamic field theory: Working memory for locations becomes more spatially precise over development, Child Dev. 74 (5) (2003) 1393-1417.

[8] C. Wilimzig, S. Schneider, G. Schöner, The time course of saccadic decision making: Dynamic field theory, Neural Netw. 19 (8) (2006) 1059-1074. 
[9] J. Fix, N. Rougier, F. Alexandre, A dynamic neural field approach to the covert and overt deployment of spatial attention, Cogn. Comput. 3 (1) (2011) 279-293.

[10] F. Ferreira, W. Erlhagen, E. Sousa, L. Louro, E. Bicho, Learning a musical sequence by observation: A robotics implementation of a dynamic neural field model, in: 2014 Joint IEEE International Conferences on Development and Learning and Epigenetic Robotics, ICDL-Epirob, IEEE, 2014, pp. 157-162.

[11] W. Erlhagen, E. Bicho, The dynamic neural field approach to cognitive robotics, J. Neural Eng. 3 (3) (2006) R36.

[12] G. Schöner, Dynamical Systems Approaches to Cognition, Cambridge University Press, Cambridge, UK, 2008, pp. 101-126.

[13] C.D. Brody, R. Romo, A. Kepecs, Basic mechanisms for graded persistent activity: discrete attractors, continuous attractors, and dynamic representations, Curr. Opin. Neurobiol. 13 (2) (2003) 204-211.

[14] S.-i. Amari, Dynamics of pattern formation in lateral-inhibition type neural fields, Biol. Cybernet. 27 (2) (1977) 77-87.

[15] R. Almeida, J. Barbosa, A. Compte, Neural circuit basis of visuo-spatial working memory precision: a computational and behavioral study, J. Neurophysiol. 114 (3) (2015) 1806-1818.

[16] C. Faubel, G. Schöner, Learning to recognize objects on the fly: a neurally based dynamic field approach, Neural Netw. 21 (4) (2008) 562-576.

[17] C.R. Laing, W.C. Troy, B. Gutkin, G.B. Ermentrout, Multiple bumps in a neuronal model of working memory, SIAM J. Appl. Math. 63 (1) (2002) 62-97.

[18] C.R. Laing, W.C. Troy, Two-bump solutions of Amari-type models of neuronal pattern formation, Physica D 178 (3) (2003) 190-218.

[19] S.-i. Amari, Topographic organization of nerve fields, Bull. Math. Biol. 42 (3) (1980) 339-364.

[20] W.-C.C. Chan, S.-S. Lin, Existence and stability of multibump solutions of an integral-differential equation, Int. J. Bifurcation Chaos 17 (11) (2007) 4099-4115.
[21] S. Coombes, G.J. Lord, M.R. Owen, Waves and bumps in neuronal networks with axo-dendritic synaptic interactions, Physica D 178 (3) (2003) 219-241.

[22] B.S. Gutkin, G.B. Ermentrout, J. O’Sullivan, Layer 3 patchy recurrent excitatory connections may determine the spatial organization of sustained activity in the primate prefrontal cortex, Neurocomputing 32 (2000) 391-400.

[23] J.A. Murdock, F. Botelho, J.E. Jamison, Persistence of spatial patterns produced by neural field equations, Physica D 215 (2) (2006) 106-116.

[24] Y. Guo, C.C. Chow, Existence and stability of standing pulses in neural networks: I. Existence, SIAM J. Appl. Dyn. Syst. 4 (2) (2005) 217-248.

[25] MATLAB, version 7.10.0 (r2010a) (2010).

[26] S. Kubota, K. Hamaguchi, K. Aihara, Local excitation solutions in onedimensional neural fields by external input stimuli, Neural Comput. Appl. 18 (6) (2009) 591-602.

[27] S.E. Folias, P.C. Bressloff, Breathing pulses in an excitatory neural network, SIAM J. Appl. Dyn. Syst. 3 (3) (2004) 378-407.

[28] J.C. Polking, dfield and pplane software, 1997-2003. http://math.rice.edu/ $\sim$ dfield.

[29] W. Erlhagen, A. Bastian, D. Jancke, A. Riehle, G. Schöner, The distribution of neuronal population activation (dpa) as a tool to study interaction and integration in cortical representations, J. Neurosci. Methods 94 (1) (1999) 53-66.

[30] J.S. Johnson, J.P. Spencer, G. Schöner, A layered neural architecture for the consolidation, maintenance, and updating of representations in visual working memory, Brain Res. 1299 (2009) 17-32.

[31] X.-J. Wang, Synaptic reverberation underlying mnemonic persistent activity, Trends Neurosci. 24 (8) (2001) 455-463.

[32] E. Bicho, L. Louro, W. Erlhagen, Integrating verbal and nonverbal communication in a dynamic neural field architecture for human-robot interaction, Front. Neurorobotics 4 (2010).

[33] S. Carroll, K. Josić, Z.P. Kilpatrick, Encoding certainty in bump attractors, J. Comput. Neurosci. 37 (1) (2014) 29-48. 